\title{
Computer Assisted Proof for Normally Hyperbolic Invariant Manifolds
}

\author{
Maciej J. Capiński \\ Faculty of Applied Mathematics, AGH University of Science and Technology, al. \\ Mickiewicza 30, 30-059 Kraków, Poland \\ E-mail: mcapinsk@agh.edu.pl
}

\section{Carles Simó}

Departament de Matemàtica Aplicada i Anàlisi, Universitat de Barcelona, Gran Via 585, 08007 Barcelona, Spain

E-mail: carles@maia.ub.es

\begin{abstract}
We present a topological proof of the existence of a normally hyperbolic invariant manifold for maps. In our approach we do not require that the map is a perturbation of some other map for which we already have an invariant manifold. But a non-rigorous, good enough, guess is necessary. The required assumptions are formulated in a way which allows for rigorous computer assisted verification. We apply our method for a driven logistic map, for which non-rigorous numerical simulation in plain double precision suggests the existence of a chaotic attractor. We prove that this numerical evidence is false and that the attractor is a normally hyperbolic invariant curve.
\end{abstract}




\section{Introduction}

In this paper we give a proof of existence of normally hyperbolic invariant manifolds for maps. The construction is performed in the state space of the map. Assumptions needed for the proof are of twofold nature. First we require topological conditions which follow from suitable alignment of the coordinates (these are the so called covering relations). Next we require that our map satisfies cone conditions. The aim of the paper though is not to produce yet another proof of the normally hyperbolic invariant manifold theorem. Our aim is to produce a tool that can be applied in rigorous-computer-assisted proofs. To show the strength of our approach we apply our theorem to a driven logistic map introduced in [2]. The considered map is such that standard numerical simulation gives evidence of a chaotic attractor. The example is a demonstration of the fact that one has to be careful with the arithmetics in simulations, since the numerical evidence of an attractor is false. The map in fact possesses a normally hyperbolic invariant curve. This is apparent when simulations are performed using multiple precision computations. The strength of our method lies in the fact that even for such an example, which defeats standard numerical simulations, we are able to produce a rigorous proof of existence of a normally hyperbolic invariant curve.

The approach to normally hyperbolic manifolds presented here is in the spirit of [3] and [6]. In [3] a topological proof of existence of invariant sets with normally hyperbolic type properties is given. In [6] the result is extended to prove normally hyperbolic invariant manifolds. In both cases the proofs relied on assumptions that the first iterate of the map is well aligned with the stable and unstable manifolds. Similar approach was also used in [4] to give a proof of existence of a center manifold. The result in [4] is for ODEs and relies also on the fact that hyperbolic dynamics is uniform. The main difference between our paper and results mentioned above is that we assume that hyperbolic expansion and contraction aligns with the tangent spaces of the invariant manifolds after a suitable (possibly large) number of iterates of the map. This setting is more general, and also more typical for normal hyperbolicity.

The paper is organized as follows. Section 2 introduces basic notations used throughout the paper and provides a setup and an outline of our problem. Section 3 contains a geometric construction of a normally hyperbolic manifold. We first give a construction of a "center-stable" manifold (the term "center-stable" refers to the normally hyperbolic invariant manifold union its associated stable manifold; analogous terminology is used by us for the "center-unstable" manifold). A center-unstable manifold is obtained using a mirror construction to the center-unstable manifold, by considering the inverse map. The intersection of the center-stable and center-unstable manifolds gives us the normally hyperbolic invariant manifold. In Section 4 we show how to verify assumptions of our theorems using local bounds on derivatives of the map. In Section 5 we present our example of the driven logistic map and apply our method to it. 


\section{Setup}

We start by writing out some basic notations which we shall use throughout the paper. A notation $B_{i}(q, r)$ will stand for a ball of radius $r$ centered at $q$ in $\mathbb{R}^{i}$. We will also use a notation $B_{i}=B_{i}(0,1)$. For a set $A$ we will denote by $\bar{A}$ its closure, by int $A$ its interior and by $\partial A$ its boundary. For a function $f$ we will use a notation $\operatorname{dom}(f)$ to denote its domain. For points $p=(x, y)$ we shall use notation $\pi_{x}(p), \pi_{y}(p)$ to denote the projection onto the $x$ and $y$ coordinates respectively.

We now introduce the setup of our problem. Let $D$ and $\mathcal{U}$ be open subsets in $\mathbb{R}^{n}$ such that $D \subset \mathcal{U}$. Let

$$
f: \mathcal{U} \rightarrow \mathcal{U}
$$

be a diffeomorphism. Let $u, s, c \in \mathbb{N}$ be such that $u+s+c=n$. We assume that there exist a diffeomorphism

$$
\phi: \mathcal{U} \rightarrow \phi(\mathcal{U}) \subset \mathbb{R}^{u} \times \mathbb{R}^{s} \times \Lambda
$$

such that $\phi(\operatorname{cl} D)=D_{\phi}:=\bar{B}_{u} \times \bar{B}_{s} \times \Lambda$, and $\Lambda$ is a compact $c$ dimensional manifold without boundary. We define $f_{\phi}: D_{\phi} \rightarrow \mathbb{R}^{u} \times \mathbb{R}^{s} \times \Lambda$ as

$$
f_{\phi}=\phi \circ f \circ \phi^{-1} \text {. }
$$

We assume that there exists a finite covering $\left\{U_{i}\right\}_{i \in I}$ of $\Lambda$ and an atlas

$$
\eta_{i}: \bar{U}_{i} \rightarrow \bar{B}_{c} .
$$

Throughout the work we will use a notation

$$
\mathbf{B}=\bar{B}_{u} \times \bar{B}_{s} \times \bar{B}_{c} .
$$

For $i, j \in I$ we consider local maps $f_{j i}: \mathbf{B} \supset \operatorname{dom}\left(f_{i j}\right) \rightarrow \mathbb{R}^{u} \times \mathbb{R}^{s} \times \bar{B}_{c}$ defined as

$$
\begin{aligned}
& f_{i j}:=\tilde{\eta}_{j} \circ f_{\phi} \circ \tilde{\eta}_{i}^{-1}, \\
& \tilde{\eta}_{i}:=\left(\mathrm{id}, \mathrm{id}, \eta_{i}\right) \quad \text { for } i \in I .
\end{aligned}
$$

Note that the domain of $f_{i j}$ can be empty, and will usually be smaller than $\mathbf{B}$. The following graph depicts the above defined functions and their mutual relations.

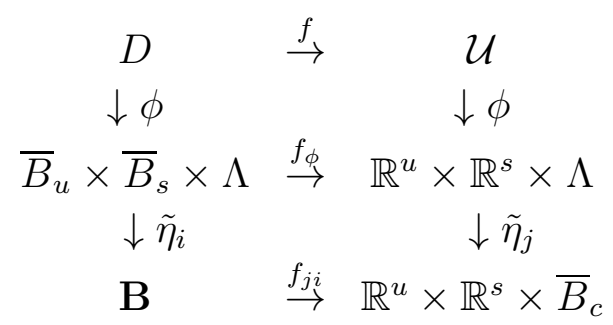

Our task in this paper will be to find a normally hyperbolic invariant manifold, together with its stable and unstable manifolds within the set $D$.

We will use the following notations for our coordinates: $x \in \mathbb{R}^{u}, y \in \mathbb{R}^{s}, \theta \in \bar{B}_{c}$, $\lambda \in \Lambda$. The coordinate $x$ will play the role of a globally unstable direction, and the coordinate $y$ will play the role of a stable direction for the map $f_{\phi}$ (hence the superscripts 
$u$ and $s$, which stand for "unstable" and "stable" respectively). The coordinate $\lambda$ will play the role of the central direction, in which the global dynamics is weaker than in the stable and unstable coordinates. The notation $\theta$ will also be used for the central direction, but it will be reserved to denote the central coordinate in the local coordinates; i.e. $\theta=\eta_{i}(\lambda)$ for some $\lambda \in \Lambda$ and $i \in I$.

\section{Geometric approach to invariant manifolds}

In this section we give the construction of a normally hyperbolic invariant manifold. The construction is performed in the state space of our map. It is based on the assumptions of covering relations and cone conditions. We first give an introduction to these tools in Section 3.1. In Section 3.2 we formulate our assumptions on the map in terms of covering relations and cone conditions, which will imply the existence of a normally hyperbolic manifold. In Section 3.3 we show how to construct a center-stable manifold of our map. The construction of a center-unstable manifold follows from a mirror argument. The intersection of center-stable and center-unstable manifolds gives us a $C^{0}$ normally hyperbolic invariant manifold. Let us write explicitly that for a normally hyperbolic manifold which does not have an associated stable manifold, the center-stable manifold will be the the normally hyperbolic manifold itself. Analogous statement holds also for center-unstable manifolds.

\subsection{Covering relations and cones}

Covering relations are topological tools used for proofs of nontrivial symbolic dynamics of dynamical systems. The method is based on the Brouwer fixed point index, and the setting is such that it allows for rigorous numerical verification. The method has been applied in computer assisted proofs for the Hénon map, Rössler equations [19], [6], Lorenz equations [8], Chua circuit [7] or Kuramoto-Shivashinsky ODE [18], amongst others. The method is based on singling out a number of regions, called h-sets, which have hyperbolic type properties. Using these properties one can find orbits of the system, which shadow the h-sets along their trajectories. The method of covering relations relies on the system having expanding and contracting coordinates. In this section we generalize covering relations to include also a central direction. The setup is similar to that of [3], [5], but has been simplified. Our proofs are now simpler and based only on continuity arguments. They no longer require the use of degree theory, with little loss of generality.

For any $p=(x, y, \theta) \in \mathbf{B}$ and $r_{u}, r_{s}, r_{c}>0$ we introduce a notation

$$
N\left(p, r_{u}, r_{s}, r_{c}\right):=\bar{B}_{u}\left(x, r_{u}\right) \times \bar{B}_{s}\left(y, r_{s}\right) \times \bar{B}_{c}\left(\theta, r_{c}\right) .
$$

We define

$$
\begin{aligned}
N^{-} & =N^{-}\left(p, r_{u}, r_{s}, r_{c}\right):=\partial \bar{B}_{u}\left(x, r_{u}\right) \times \bar{B}_{s}\left(y, r_{s}\right) \times \bar{B}_{c}\left(\theta, r_{c}\right) \\
N^{+} & =N^{+}\left(p, r_{u}, r_{s}, r_{c}\right) \\
& :=\bar{B}_{u}\left(x, r_{u}\right) \times\left(\left(\mathbb{R}^{s} \times \mathbb{R}^{c}\right) \backslash\left(B_{s}\left(y, r_{s}\right) \times B_{c}\left(\theta, r_{c}\right)\right)\right) .
\end{aligned}
$$




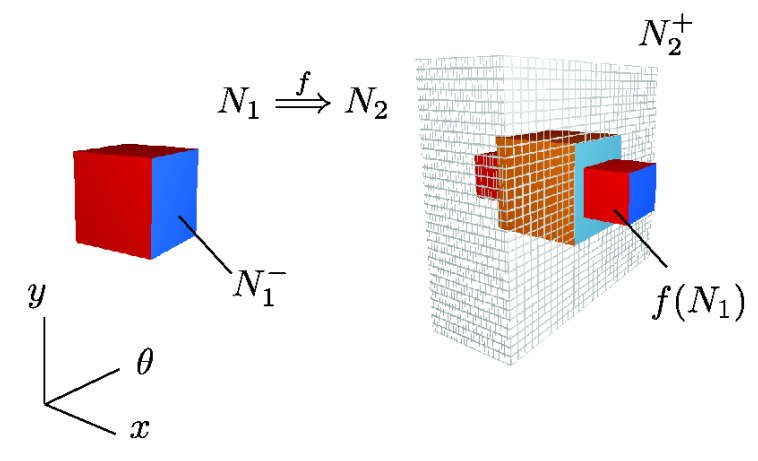

Figure 1. A ch-set $N_{1}$ covering a ch-set $N_{2}$.

We assume that all boxes $N$ which we are going to consider here are contained in $\mathbf{B}$. We will refer to a box $N$ as a ch-set (center-hyperbolic set) centered at $p$.

In following arguments we shall often consider different ch-sets. To keep better track of our notations and to make our arguments more transparent we shall stick to a convention that for two ch-sets $N_{1}, N_{2}$ centered respectively at $p_{1}=\left(x_{1}, y_{1}, \theta_{1}\right)$ and $p_{2}=\left(x_{2}, y_{2}, \theta_{2}\right)$ we shall write

$$
N_{i}=N_{i}\left(p_{i}, r_{u}^{i}, r_{s}^{i}, r_{c}^{i}\right):=\bar{B}_{u}^{i}\left(x_{i}, r_{u}^{i}\right) \times \bar{B}_{s}^{i}\left(y_{i}, r_{s}^{i}\right) \times \bar{B}_{c}^{i}\left(\theta_{i}, r_{c}^{i}\right) \quad \text { for } i=1,2 .
$$

Definition 1. Let $g: \mathbf{B} \rightarrow \mathbb{R}^{u} \times \mathbb{R}^{s} \times \bar{B}_{c}$ be a continuous function. Let $p_{i}=\left(x_{i}, y_{i}, \theta_{i}\right)$ for $i=1,2$ and let $N_{1}, N_{2}$ be two ch-sets in $\mathbf{B}$ centered at $p_{1}$ and $p_{2}$ respectively. We say that $N_{1} g$-covers $N_{2}$ if

$$
\begin{aligned}
& g\left(p_{1}\right) \in \operatorname{int}\left(N_{2}\right), \\
& \pi_{x}\left(g\left(N_{1}^{-}\right)\right) \cap \bar{B}_{u}^{2}\left(x_{2}, r_{u}^{2}\right)=\emptyset, \\
& g\left(N_{1}\right) \cap N_{2}^{+}=\emptyset .
\end{aligned}
$$

In such case we shall write $N_{1} \stackrel{g}{\Longrightarrow} N_{2}$.

Remark 2. Definition 1 is a simplified definition of a covering relation. More general versions can be found in [8], 9], [19] in the setting of hyperbolicity, or in [3], [5] in a setting when additionally a central direction is included.

For $\gamma=(a, b, c) \in \mathbb{R}^{3}$, and $q=(x, y, \theta) \in \mathbb{R}^{u} \times \mathbb{R}^{s} \times \mathbb{R}^{c}$ we define

$$
\begin{aligned}
& Q_{\gamma}: \mathbb{R}^{u} \times \mathbb{R}^{s} \times \mathbb{R}^{c} \rightarrow \mathbb{R} \\
& Q_{\gamma}(q):=a\|x\|^{2}+b\|y\|^{2}+c\|\theta\|^{2} .
\end{aligned}
$$

If $a>0 b, c<0$, then for $p \in \mathbb{R}^{u} \times \mathbb{R}^{s} \times \mathbb{R}^{c}$ we will refer to

$$
C(p, \gamma):=\left\{q: Q_{\gamma}(p-q) \geq 0\right\}
$$

as a horizontal cone centered at $p$ (see Figure 2).

Definition 3. Let $N$ be a ch-set and $\gamma=(a, b, c)$ be such that $a>0, b, c<0$. We will refer to a pair $(N, \gamma)$ as a ch-set with cones. 
Definition 4. Let $(N, \gamma)=\left(N\left((x, y, \theta), r_{u}, r_{s}, r_{c}\right), \gamma\right)$ be a ch-set with cones. A continuous function $\mathbf{h}: \bar{B}_{u}\left(x, r_{u}\right) \rightarrow N$ is called a horizontal disc in $(N, \gamma)$, iff $\pi_{x} \mathbf{h}(x)=x$ and for any $x^{*}, x^{* *} \in \bar{B}_{u}\left(x, r_{u}\right)$,

$$
Q_{\gamma}\left(\mathbf{h}\left(x^{*}\right)-\mathbf{h}\left(x^{* *}\right)\right) \geq 0,
$$

Lemma 5. Let $N_{i}=N_{i}\left(\left(x_{i}, y_{i}, \theta_{i}\right), r_{u}^{i}, r_{s}^{i}, r_{c}^{i}\right)$ for $i=1,2$ and let $\left(N_{1}, \gamma_{1}\right),\left(N_{2}, \gamma_{2}\right)$ be two ch-sets with cones. Assume that

$$
N_{1} \stackrel{g}{\Longrightarrow} N_{2}
$$

and that for any $q^{*}, q^{* *} \in N_{1}$ such that $q^{*} \neq q^{* *}$ and $Q_{\gamma_{1}}\left(q^{*}-q^{* *}\right) \geq 0$ we have

$$
Q_{\gamma_{2}}\left(g\left(q^{*}\right)-g\left(q^{* *}\right)\right)>0 .
$$

If $\mathbf{h}_{1}$ is a horizontal disc in $\left(N_{1}, \gamma_{1}\right)$ then there exists a horizontal disc $\mathbf{h}_{2}$ in $\left(N_{2}, \gamma_{2}\right)$ such that $g\left(\mathbf{h}_{1}\left(\bar{B}_{u}^{1}\left(x_{1}, r_{u}^{1}\right)\right)\right) \cap N_{2}=\mathbf{h}_{2}\left(\bar{B}_{u}^{2}\left(x_{2}, r_{u}^{2}\right)\right)$.

Proof. Without loss of generality we assume that $p_{1}=p_{2}=0$ and that $r_{\kappa}^{i}=1$ for $i=1,2$ and $\kappa \in\{u, s, c\}$. In other words we assume that for $i=1,2$

$$
N_{i}=\bar{B}_{u}^{i} \times \bar{B}_{s}^{i} \times \bar{B}_{c}^{i}=\bar{B}_{u}(0,1) \times \bar{B}_{s}(0,1) \times \bar{B}_{c}(0,1) .
$$

Let $\gamma_{i}=\left(a_{i}, b_{i}, c_{i}\right)$ for $i=1,2$ and let $\mathbf{h}$ be any horizontal disc in $N_{1}$. Then by (4), (5) and (77) for $x^{*}, x^{* *} \in \bar{B}_{u}^{1}, x^{*} \neq x^{* *}$

$$
a_{2}\left\|\pi_{x} g\left(\mathbf{h}\left(x^{*}\right)\right)-\pi_{x} g\left(\mathbf{h}\left(x^{* *}\right)\right)\right\|^{2} \geq Q_{\gamma_{2}}\left(g\left(\mathbf{h}\left(x^{*}\right)\right)-g\left(\mathbf{h}\left(x^{* *}\right)\right)\right)>0,
$$

which means that $\pi_{x} \circ g \circ \mathbf{h}$ is a monomorphism.

Using a notation $\mathbf{h}_{1}(x)=\left(x, h_{1}(x)\right) \in \bar{B}_{u}^{i} \times\left(\bar{B}_{s}^{i} \times \bar{B}_{c}^{i}\right)$, for $\alpha \in[0,1]$, we define a family of horizontal discs $\mathbf{h}_{\alpha}(x)=\left(x, \alpha h_{1}(x)\right)$. Let $F_{\alpha}: \bar{B}_{u}^{1} \rightarrow \mathbb{R}^{u}$ be a continuous family of functions defined as

$$
F_{\alpha}(x):=\pi_{x} \circ g \circ \mathbf{h}_{\alpha}(x) .
$$

We shall show that $\bar{B}_{u}^{2} \subset F_{1}\left(B_{u}^{1}\right)$. Functions $F_{\alpha}$ are monomorphisms, hence sets $A_{\alpha}:=F_{\alpha}\left(B_{u}^{1}\right)$ are homeomorphic to balls in $\mathbb{R}^{u}$; moreover $\partial A_{\alpha}=F_{\alpha}\left(\partial B_{u}^{1}\right)$. By Definition 4 of a horizontal disc, $\mathbf{h}_{\alpha}\left(\partial B_{u}^{1}\right) \subset N_{1}^{-}$. From assumption ([6) ), by conditions (1), (2)

$$
\begin{aligned}
& \pi_{x} g(0) \in \bar{B}_{u}^{2}, \\
& \partial A_{\alpha} \cap \bar{B}_{u}^{2} \subset F_{\alpha}\left(N_{1}^{-}\right) \cap \bar{B}_{u}^{2}=\emptyset .
\end{aligned}
$$

From the fact that $0 \in B_{u}^{1}$

$$
F_{0}(0) \in F_{0}\left(B_{u}^{1}\right)=A_{0} .
$$

Since $\mathbf{h}_{0}(0)=0$, by (9)

$$
F_{0}(0)=\pi_{x} \circ g \circ \mathbf{h}_{0}(0)=\pi_{x} g(0) \in \bar{B}_{u}^{2},
$$

From (11), (12) follows that $A_{0} \cap \bar{B}_{u}^{2} \neq \emptyset$. This by (10) implies that $\bar{B}_{u}^{2} \subset A_{0}$. By continuity of $F_{\alpha}$ with respect to $\alpha$ this means that $\bar{B}_{u}^{2} \subset A_{\alpha}$ for all $\alpha \in[0,1]$. In particular $\bar{B}_{u}^{2} \subset A_{1}=F_{1}\left(B_{u}^{1}\right)$. 


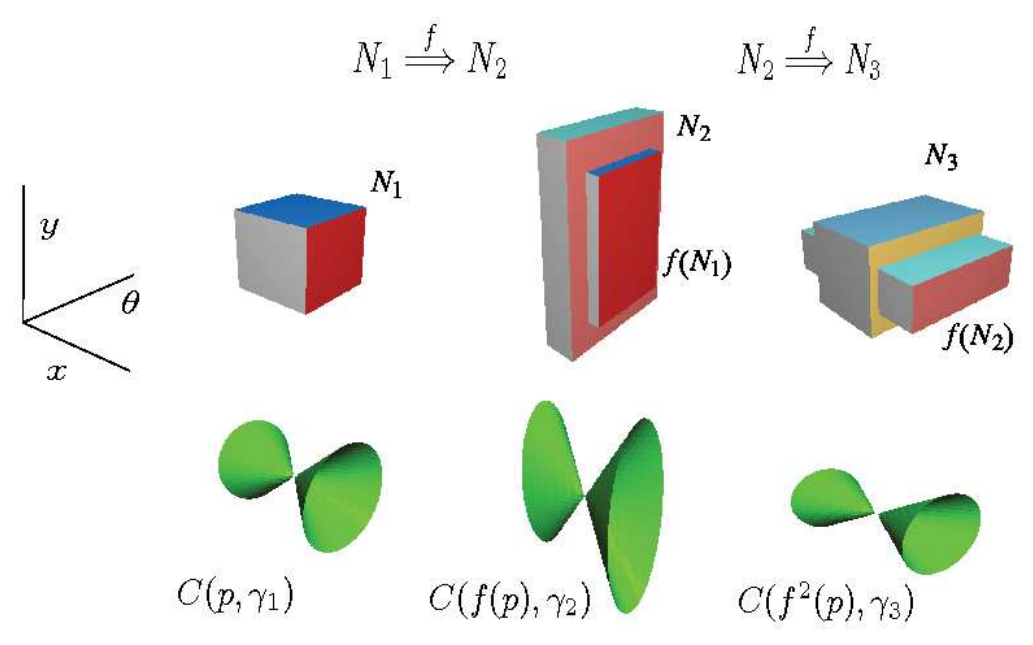

Figure 2. Covering relations for two iterates of a map $f$. For the second iterate of the map the coordinate $x$ is expanding and $y$ is contracting (for the first iterate of $f$ they are not). The fact that expansion in $x$ is stronger than expansion in $\theta$ is visible from the fact that the cones $C\left(f^{2}(p), \gamma_{3}\right)$ are "tighter" than cones $C\left(p, \gamma_{1}\right)$.

Since $F_{1}$ is a monomorphism and $\bar{B}_{u}^{2} \subset F_{1}\left(B_{u}^{1}\right)$, for any $v \in \bar{B}_{u}^{2}$ there exists a unique $x=x(v) \in B_{u}^{1}$ such that $F_{1}(x)=v$. We define $\mathbf{h}_{2}(v)=\left(v, h_{2}(v)\right):=\left(v, \pi_{y, \theta} \circ g \circ \mathbf{h}_{1}(x(v))\right)$. For any $v^{*} \neq v^{* *}, v^{*}, v^{* *} \in \bar{B}_{u}^{2}$, by (15) and (17) we have

$$
\begin{aligned}
Q_{\gamma_{2}}\left(\mathbf{h}_{2}\left(v^{*}\right)-\mathbf{h}_{2}\left(v^{*}\right)\right) & =Q_{\gamma_{2}}\left(g \circ \mathbf{h}_{1}\left(x\left(v^{*}\right)\right)-g \circ \mathbf{h}_{1}\left(x\left(v^{* *}\right)\right)\right) \\
& >Q_{\gamma_{1}}\left(\mathbf{h}_{1}\left(x\left(v^{*}\right)\right)-\mathbf{h}_{1}\left(x\left(v^{* *}\right)\right)\right) \\
& >0 .
\end{aligned}
$$

Since $Q_{\gamma_{2}}\left(\mathbf{h}_{2}\left(v^{*}\right)-\mathbf{h}_{2}\left(v^{* *}\right)\right)>0$

$$
\begin{aligned}
& a_{2}\left\|v^{*}-v^{* *}\right\| \\
& >-b_{2}\left\|\pi_{y}\left(h_{2}\left(v^{*}\right)-h_{2}\left(v^{* *}\right)\right)\right\|^{2}-c_{2}\left\|\pi_{\theta}\left(h_{2}\left(v^{*}\right)-h_{2}\left(v^{* *}\right)\right)\right\|^{2} \\
& \geq \min \left(-b_{2},-c_{2}\right)\left\|h_{2}\left(v^{*}\right)-h_{2}\left(v^{* *}\right)\right\|^{2},
\end{aligned}
$$

and therefore $\mathbf{h}_{2}$ is continuous.

Remark 6. Let us note that since we have freedom of choice of the radii $r_{u}, r_{s}$ and $r_{c}$ it is not necessary for $x$ to be expanding, $y$ to be contracting and $\theta$ to have weaker dynamics for each single iterate of the map. In Figure 2 we have a sketch of a situation in which $x$ becomes expanding and $y$ contracting after a second iterate. In Figure 2 the coordinate $\theta$ is expanding. It will turn out that such a scenario is acceptable for us and can be dealt with by increasing $r_{c}$ for successive iterates.

\subsection{Covering relations and cone conditions for normal hyperbolicity}

In this section we formulate our assumptions which will imply the existence of a normally hyperbolic manifold. The assumptions are in terms of covering relations 
and cones and are in the spirit of [5]. There are two major differences though. The first is that assumptions used in [5] required the system to have uniform expansion and uniform contraction for the first iterate of the map. Here we set up our coordinates in the directions of global contraction and global expansion. In the setting of normal hyperbolicity the coordinates of global contraction and expansion need not be contracting and expanding for the first iterates of the map. What is important is that they dominate after a sufficiently large numbers of iterates, in other words, that the Lyapunov exponents are negative or positive, respectively. We set up our assumptions so that they allow for such setting. The second difference is that our setup has been significantly simplified with comparison to [5]. This resulted in a slight loss of generality (we do not formulate our assumptions in terms of vector bundles as in [5]) but we need to consider fewer assumptions.

Let $1>R>\rho, r>0$. Assume that there exists a finite sequence of points $\boldsymbol{\lambda}_{k} \in \Lambda, k \in \mathbb{N}$ such that for any $k$ the set $I(k)=\left\{i: B_{c}\left(\eta_{i}\left(\boldsymbol{\lambda}_{k}\right), \rho\right) \subset B_{c}(0, R)\right\}$ is not empty. What is more, assume that there exists a set $J \subset\{(i, k) \mid i \in I(k)\}$ such that $\Lambda \subset \bigcup_{(i, k) \in J} \eta_{i}^{-1}\left(B_{c}\left(\eta_{i}\left(\boldsymbol{\lambda}_{k}\right), \rho\right)\right)$. For points $(i, k) \in J$ we define sets

$$
M_{i, k}:=\bar{B}_{u}(0, r) \times \bar{B}_{s}(0, r) \times \bar{B}_{c}\left(\eta_{i}\left(\boldsymbol{\lambda}_{k}\right), \rho\right) .
$$

We will need to assume that the points $\boldsymbol{\lambda}_{k}$ are sufficiently close to each other. We will also need to assume that $R$ and $\rho$ are sufficiently large in comparison to $r$. This is summarized in Assumption 7. The idea behind it is demonstrated in Figure 3, which might provide some intuition.

Assumption 7. Let $\mathbf{m}>1$ and let $\gamma_{0}=\left(\mathbf{a}_{0}, \mathbf{b}_{0}, \mathbf{c}_{0}\right) \in \mathbb{R}^{3}, \gamma_{1}=\left(\mathbf{a}_{1}, \mathbf{b}_{1}, \mathbf{c}_{1}\right) \in \mathbb{R}^{3}$ satisfy $\mathbf{a}_{m}>0, \mathbf{b}_{m}, \mathbf{c}_{m}<0$ for $m=1,2$. Let us also define a set $M \subset \mathbf{B}$ as

$$
M:=\bar{B}_{u}(0, r) \times \bar{B}_{s}(0, r) \times \bar{B}_{c} \text {. }
$$

We assume that for any horizontal disc $\mathbf{h}$ in a ch-set with cones $\left(M, \gamma_{1}\right)$ and for any $i \in I$ there exists $(\iota, \kappa) \in J$ such that $\mathbf{h}\left(B_{u}(0, r)\right) \subset \operatorname{dom}\left(\tilde{\eta}_{\iota} \circ \tilde{\eta}_{i}^{-1}\right)$. In addition we assume that for any $q^{*}, q^{* *}$ in $\operatorname{dom}\left(\tilde{\eta}_{\iota} \circ \tilde{\eta}_{i}^{-1}\right)$ such that $Q_{\gamma_{1}}\left(q^{*}-q^{* *}\right)>0$ we have

$$
Q_{\gamma_{0}}\left(\tilde{\eta}_{\iota} \circ \tilde{\eta}_{i}^{-1}\left(q^{*}\right)-\tilde{\eta}_{\iota} \circ \tilde{\eta}_{i}^{-1}\left(q^{* *}\right)\right)>\mathbf{m} Q_{\gamma_{1}}\left(q^{*}-q^{* *}\right),
$$

and

$$
\mathbf{h}^{\prime}:=\tilde{\eta}_{\iota} \circ \tilde{\eta}_{i}^{-1} \circ \mathbf{h}_{\mid B_{u}(0, r)} \quad \text { is a horizontal disc in }\left(M_{\iota, \kappa}, \boldsymbol{\gamma}_{0}\right) \text {. }
$$

Assumption 7 ensures that for $\mathbf{h}$ in some local coordinates $\tilde{\eta}_{i}$ we can change to coordinates $\tilde{\eta}_{\iota}$ so that $\mathbf{h}^{\prime}:=\tilde{\eta}_{\iota} \circ \tilde{\eta}_{i}^{-1} \circ \mathbf{h}$ lies close to the middle of the set $M$. Assumption 7 is also discussed in Section 4.3, where conditions which imply it are given.

Remark 8. Above we use bold font for $\gamma_{i}=\left(\mathbf{a}_{i}, \mathbf{b}_{i}, \mathbf{c}_{i}\right), i=0,1$ to emphasize that these are fixed constants, and to distinguish them from other $\gamma=(a, b, c)$ in our proofs.

Definition 9. If for any $(i, k) \in J$ there exists a sequence of ch-sets with cones $\left(N_{1}, \gamma_{1}\right), \ldots,\left(N_{n}, \gamma_{n}\right)$ ( $n$ can depend on $(i, k)$ ) and a sequence $i_{0}=i, i_{1}, \ldots, i_{n} \in I$ such that

$$
M_{i, k}=: N_{0} \stackrel{f_{i_{1} i_{0}}}{\longrightarrow} N_{1} \stackrel{f_{i_{2} i_{1}}}{\longrightarrow} N_{2} \stackrel{f_{i_{3} i_{2}}}{\longrightarrow} \ldots \stackrel{f_{i_{n} i_{n}-1}}{\longrightarrow} N_{n} \stackrel{\mathrm{id}}{\Longrightarrow} M
$$




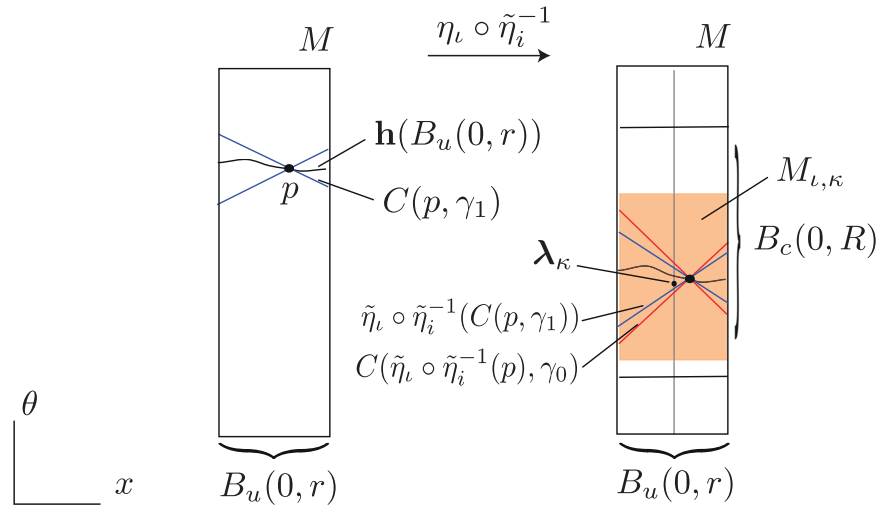

Figure 3. The change of coordinates $\tilde{\eta}_{\iota} \circ \tilde{\eta}_{i}^{-1}$, a horizontal disc $\mathbf{h}$, and the cones given by $\gamma_{0}$ and $\gamma_{1}$ in different local coordinates. Here, for simplicity, the stable coordinate is neglected $s=0$.

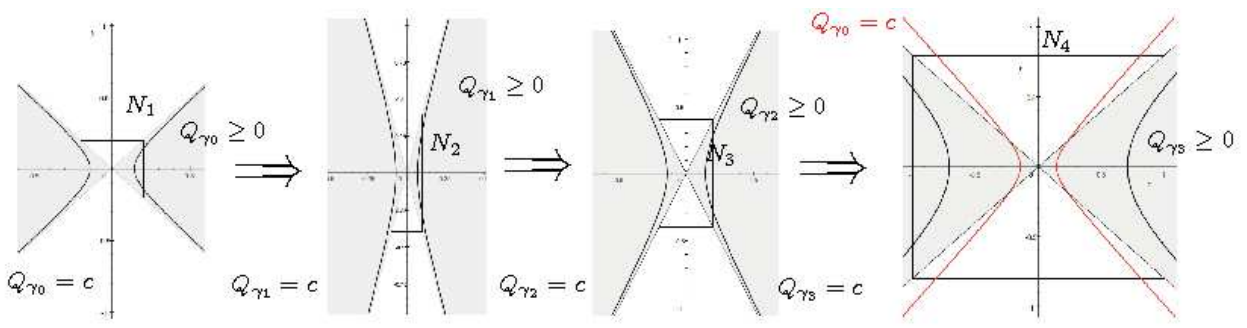

Figure 4. (see Example 10) For the first iterates of the map the ch-sets and cones are contracted in the $x$ direction. After a number of steps the expansion in $x$ starts to dominate. Note that the coordinate $\theta$ is expanding. Since expansion in $x$ is stronger than expansion in $\theta$ though, the cones eventually become more flat and their level sets $Q_{\gamma_{i}}=c$ are pulled away from the origin.

then we say that $f$ satisfies covering conditions.

If in addition for any $q_{1}, q_{2} \in N_{l-1}, q_{1} \neq q_{2}$,

$$
Q_{\gamma_{l+1}}\left(f_{i_{l+1} i_{l}}\left(q_{1}\right)-f_{i_{l+1} i_{l}}\left(q_{2}\right)\right)>Q_{\gamma_{l}}\left(q_{1}-q_{2}\right)
$$

for $l=0, \ldots, n-1$, and for $\gamma_{n}=(a, b, c)$ we have

$$
\mathbf{a}_{1}>a, \quad \frac{\mathbf{b}_{1}}{\mathbf{a}_{1}}>\frac{b}{a}, \quad \frac{\mathbf{c}_{1}}{\mathbf{a}_{1}}>\frac{c}{a},
$$

then we say that $f$ satisfies cone conditions.

Example 10. This example stands behind the pictures from Figure 4 . Consider $u=c=1$ and $s=0$. Assume that $f_{i_{1} i_{0}}=\left(A_{i j}^{1}\right)_{i, j=1,2}=\operatorname{diag}\left(\frac{1}{2}, 2\right) f_{i_{2} i_{1}}=\left(A_{i j}^{2}\right)_{i, j=1,2}=\operatorname{diag}(2,1)$, $f_{i_{2} i_{3}}=\left(A_{i j}^{3}\right)_{i, j=1,2}=\operatorname{diag}(5,2)$. Let $\gamma_{0}=(1,-1)$ and $\gamma_{1}=\left(\frac{1}{4},-\frac{3}{8}\right)$. We take ch-sets with cones $\left(N_{l}\left((0,0), r_{u}^{l}, r_{c}^{l}\right), \gamma_{l}\right)$, for $l=0,1,2,3$ with

$$
\begin{aligned}
r_{u}^{0} & =r_{c}^{0}=r, \\
r_{u}^{l} & =r_{u}^{l-1} A_{11}^{l}-\varepsilon, \\
r_{c}^{l} & =r_{c}^{l-1} A_{22}^{l}+\varepsilon,
\end{aligned}
$$




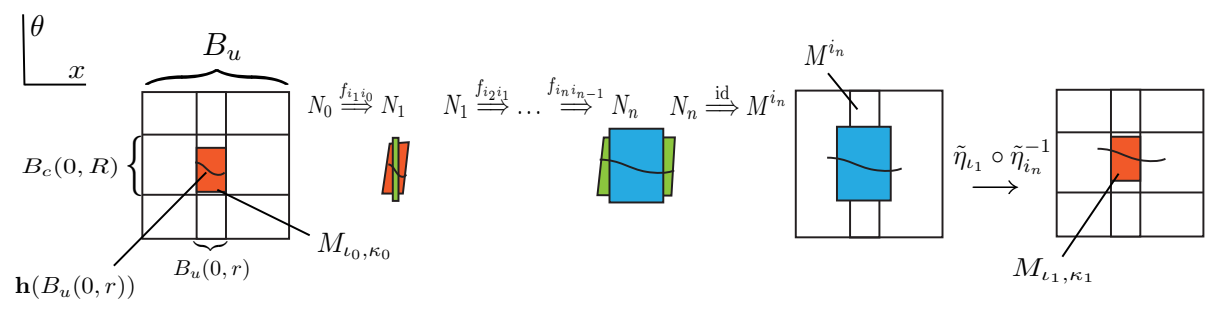

Figure 5. The sequence of covering relations from Definition 9, together with the sets $M_{\iota_{0}, \kappa_{0}}$ and $M_{\iota_{1}, \kappa_{1}}$, which are the first step of the inductive construction from the proof of Theorem [1].

$\gamma_{0}=\gamma_{0}, \gamma_{1}=\left(4 \delta,-\frac{1}{4} \delta^{-1}\right), \gamma_{2}=\left(1 \delta^{2},-\frac{1}{4} \delta^{-2}\right), \gamma_{3}=\left(\frac{1}{25} \delta^{3},-\frac{1}{16} \delta^{-3}\right)$, with $\delta=1+\varepsilon$. For sufficiently small $r$ and $\varepsilon$ we will have (16) and (17). For sufficiently small $\varepsilon$ we also have (18). Assume now that $\tilde{\eta}_{\iota} \circ \tilde{\eta}_{i_{3}}^{-1}=\operatorname{diag}\left(1,1+\frac{1}{4}\right)$. This $\tilde{\eta}_{\iota} \circ \tilde{\eta}_{i_{3}}^{-1}$ is taken just as a hypothetical example, in order to show that even when a switch to new coordinates involves an expansion in the central coordinate the Assumption 7 can easily be satisfied. We have

$$
\begin{aligned}
Q_{\gamma_{0}}\left(\tilde{\eta}_{\iota} \circ \tilde{\eta}_{i_{3}}^{-1}(x, y)\right) & =x^{2}-\frac{5}{4} \theta^{2} \\
& =4\left(\frac{1}{4} x^{2}-\frac{5}{16} \theta^{2}\right) \\
& \geq 4\left(\frac{1}{4} x^{2}-\frac{3}{8} \theta^{2}\right) \\
& =4 Q_{\gamma_{1}}((x, y))
\end{aligned}
$$

which means that (14) holds for $\mathbf{m}<4$.

We now introduce a notation $U \subset D_{\phi}$ for a set

$$
U:=B_{u}(0, r) \times B_{s}(0, r) \times \Lambda .
$$

The set $U$ will be the region in which we will construct an invariant manifold of points, which stay within the set $D_{\phi}$ for forward iterations of the map $f_{\phi}$.

\subsection{Existence of a normally hyperbolic manifold - Main result}

In this section we use the assumptions from Section 3.2 to obtain the existence of a normally hyperbolic invariant manifold inside of the set $U$ defined in (19). We start with a construction of the center-stable manifold. This is given in Theorem 11, The existence of an center-unstable manifold follows from mirror arguments for the inverse map. The normally hyperbolic manifold is obtained by intersecting the center-stable and center-unstable manifolds. This is done in Theorem 13 .

Theorem 11. If $f$ satisfies cone conditions then there exists a continuous monomorphism $V: B_{s}(0, r) \times \Lambda \rightarrow U$ such that

(i) $\pi_{y} V(y, \lambda)=y, \pi_{\lambda} V(y, \lambda)=\lambda$, 
(ii) for any $(y, \lambda) \in B_{s}(0, r) \times \Lambda$ and any $n \in \mathbb{N}$

$$
f_{\phi}^{n}(V(y, \lambda)) \in D_{\phi}
$$

(iii) for any $q \in U$ such that $f_{\phi}^{n}(q) \in D_{\phi}$ for all $n \in \mathbb{N}$, there exists a $(y, \lambda) \in B_{s}(0, r) \times \Lambda$ such that $q=V(y, \lambda)$,

(iv) if $\lambda^{*}, \lambda^{* *} \in \eta_{i}^{-1}\left(B_{c}\left(\eta_{i}\left(\boldsymbol{\lambda}_{k}\right), \rho\right)\right)$ for some $(i, k) \in J$ then for any $y^{*}, y^{* *} \in \bar{B}_{s}(0, r)$ such that $\left(\lambda^{*}, y^{*}\right) \neq\left(\lambda^{* *}, y^{* *}\right)$

$$
Q_{\gamma_{0}}\left(\tilde{\eta}_{i} \circ V\left(y^{*}, \lambda^{*}\right)-\tilde{\eta}_{i} \circ V\left(y^{* *}, \lambda^{* *}\right)\right)<0 .
$$

Proof. We take any $y_{0} \in B_{s}(0, r), \lambda_{0} \in \Lambda$ and $\left(\iota_{0}, \kappa_{0}\right) \in J$ such that $\lambda_{0} \in \eta_{\iota_{0}}^{-1}\left(B_{c}\left(\boldsymbol{\lambda}_{\kappa_{0}}, \rho\right)\right)$ and define a horizontal disc $\mathbf{h}_{0}$ in $M_{\iota_{0}, \kappa_{0}}$ as

$$
\mathbf{h}_{0}(x):=\left(x, y_{0}, \eta_{\iota_{0}}\left(\lambda_{0}\right)\right) .
$$

Since $f$ satisfies cone conditions, using assumption (16) and applying inductively Lemma 5 gives us the existence of indexes $i_{1}, \ldots, i_{n_{1}} \in I$ and of a horizontal disc $\mathbf{h}_{1}$ in $\left(M, \gamma_{n_{1}}\right)$ such that

$$
\begin{gathered}
\mathbf{h}_{1}\left(\bar{B}_{u}\right)=\left\{\tilde{\eta}_{i_{n_{1}}} \circ f_{\phi}^{n_{1}} \circ \tilde{\eta}_{\iota_{0}}^{-1}\left(\mathbf{h}_{0}(x)\right) \in M: x \in B_{u}(0, r),\right. \text { and } \\
\left.\tilde{\eta}_{i_{l}} \circ f_{\phi}^{l} \circ \tilde{\eta}_{\iota_{0}}^{-1}\left(\mathbf{h}_{1}(x)\right) \in N_{l} \text { for } l=1, \ldots, n_{1}\right\} .
\end{gathered}
$$

By (18)

$$
Q_{\gamma_{1}}\left(\mathbf{h}_{1}\left(x^{*}\right)-\mathbf{h}_{1}\left(x^{* *}\right)\right)>Q_{\gamma_{n_{1}}}\left(\mathbf{h}_{1}\left(x^{*}\right)-\mathbf{h}_{1}\left(x^{* *}\right)\right)>0,
$$

which means that $\mathbf{h}_{1}$ is a horizontal disc in $\left(M, \gamma_{1}\right)$. From (14) and (15) we know that there exists $\left(\iota_{1}, \kappa_{1}\right) \in J$ such that $\mathbf{h}^{\prime}:=\tilde{\eta}_{\iota_{1}} \circ \tilde{\eta}_{i_{n_{1}}}^{-1} \circ \mathbf{h}_{1}$ is a horizontal disc in $\left(M_{\iota_{1}, \kappa_{1}}, \gamma_{0}\right)$. This in particular means that for $\mathbf{f}_{1}:=\tilde{\eta}_{\iota_{1}} \circ f_{\phi}^{n_{1}} \circ \tilde{\eta}_{\iota_{0}}^{-1}$, there exists an $x \in B_{u}(0, r)$ for which $\mathbf{f}_{1}\left(\mathbf{h}_{1}(x)\right) \cap M_{\iota_{1}, \kappa_{1}} \neq \emptyset$. By (17) and (14), for any $x^{*} \neq x^{* *}$ such that $\mathbf{h}_{1}\left(x^{*}\right), \mathbf{h}_{1}\left(x^{* *}\right) \in \operatorname{dom}\left(\mathbf{f}_{1}\right)$

$$
Q_{\gamma_{0}}\left(\mathbf{f}_{1}\left(\mathbf{h}_{1}\left(x^{*}\right)\right)-\mathbf{f}_{1}\left(\mathbf{h}_{1}\left(x^{* *}\right)\right)\right)>\mathbf{m} Q_{\gamma_{0}}\left(\mathbf{h}_{1}\left(x^{*}\right)-\mathbf{h}_{1}\left(x^{* *}\right)\right)>0 .
$$

Repeating the above procedure inductively (starting the second step with the horizontal disc $\mathbf{h}^{\prime}$ and local coordinates given by $\tilde{\eta}_{\iota_{1}}$ ) we obtain a sequence of points $x_{s} \in B_{u}(0, r)$ and indexes $\left(\iota_{s}, \kappa_{s}\right)$ for $s \in \mathbb{N}$ such that for

$$
\mathbf{f}_{s}:=\tilde{\eta}_{\iota_{s}} \circ f_{\phi}^{n_{s}+\ldots+n_{1}} \circ \tilde{\eta}_{\iota_{0}}^{-1}
$$

we have

$$
\mathbf{f}_{w}\left(\mathbf{h}_{1}\left(x_{s}\right)\right) \in M_{\iota_{w}, \kappa_{w}} \quad \text { for } w \leq s .
$$

Since $\bar{B}_{u}(0, r)$ is compact, there exists an $x_{0}=x_{0}\left(y_{0}, \lambda_{0}\right) \in B_{u}(0, r)$ such that $\tilde{\eta}_{\iota_{s}}^{-1} \circ \mathbf{f}_{s}\left(\mathbf{h}_{1}\left(x_{0}\right)\right) \in U$ for all $s \in \mathbb{N}$. We define $V\left(y_{0}, \lambda_{0}\right):=\tilde{\eta}_{\iota_{0}}^{-1}\left(x_{0}\left(y_{0}, \lambda_{0}\right), y_{0}, \lambda_{0}\right)$. To 
see that $V$ is properly defined suppose that we have two points $x_{0}^{*} \neq x_{0}^{* *}$ such that $\tilde{\eta}_{\iota_{s}} \circ \mathbf{f}\left(\mathbf{h}_{1}\left(x_{0}^{*}\right)\right), \tilde{\eta}_{\iota_{s}} \circ \mathbf{f}\left(\mathbf{h}_{1}\left(x_{0}^{* *}\right)\right) \in U$ for all $s \in \mathbb{N}$. Then by (21) we obtain

$$
\begin{aligned}
Q_{\gamma_{0}}\left(\mathbf{f}_{s}\left(\mathbf{h}_{1}\left(x_{0}^{*}\right)\right)-\mathbf{f}_{s}\left(\mathbf{h}_{1}\left(x_{0}^{* *}\right)\right)\right) & >\mathbf{m} Q_{\gamma_{0}}\left(\mathbf{f}_{s-1}\left(\mathbf{h}_{1}\left(x_{0}^{*}\right)\right)-\mathbf{f}_{s-1}\left(\mathbf{h}_{1}\left(x_{0}^{* *}\right)\right)\right) \\
& >\ldots \\
& >\mathbf{m}^{s} Q_{\gamma_{0}}\left(\mathbf{h}_{1}\left(x_{0}^{*}\right)-\mathbf{h}_{1}\left(x_{0}^{* *}\right)\right) \\
& >0 .
\end{aligned}
$$

Since $\mathbf{m}>1$, (22) implies in particular that

$$
\left\|\pi_{x}\left(\mathbf{f}_{s}\left(\mathbf{h}_{1}\left(x_{0}^{1}\right)\right)-\mathbf{f}_{s}\left(\mathbf{h}_{1}\left(x_{0}^{2}\right)\right)\right)\right\| \rightarrow \infty \quad \text { as } \quad s \rightarrow \infty .
$$

This is impossible since $\mathbf{f}_{s}\left(\mathbf{h}_{1}\left(x_{0}^{w}\right)\right)$ is in $M_{\iota_{s}, \kappa_{s}}$, which is a subset of $\mathbf{B}$, which is a bounded.

We now need to show (20). Suppose that $V\left(y^{*}, \lambda^{*}\right), V\left(y^{* *}, \lambda^{* *}\right) \in M_{i, k}$ and $Q_{\gamma_{0}}\left(\tilde{\eta}_{i} \circ V\left(y^{*}, \lambda^{*}\right)-\tilde{\eta}_{i} \circ V\left(y^{* *}, \lambda^{* *}\right)\right) \geq 0$. Applying estimates analogous to (22) we obtain a contradiction.

Continuity of $V$ will follow from the fact that

$$
Q_{\gamma_{0}}\left(\tilde{\eta}_{\iota_{0}} \circ V\left(y^{*}, \lambda^{*}\right)-\tilde{\eta}_{\iota_{0}} \circ V\left(y^{* *}, \lambda^{* *}\right)\right)<0 .
$$

Since $\boldsymbol{\gamma}_{0}=\left(\mathbf{a}_{0}, \mathbf{b}_{0}, \mathbf{c}_{0}\right)$ with $\mathbf{a}_{0}>0$ and $\mathbf{b}_{0}, \mathbf{c}_{0}<0$ (23) gives

$$
\begin{aligned}
0> & Q_{\gamma_{0}}\left(\tilde{\eta}_{\iota_{0}} \circ V\left(y^{*}, \lambda^{*}\right)-\tilde{\eta}_{\iota_{0}} \circ V\left(y^{* *}, \lambda^{* *}\right)\right) \\
= & \mathbf{a}_{0}\left\|\pi_{x} V\left(y^{*}, \lambda^{*}\right)-\pi_{x} V\left(y^{* *}, \lambda^{* *}\right)\right\|^{2}+\mathbf{b}_{0}\left\|y^{*}-y^{* *}\right\|^{2} \\
& +\mathbf{c}_{0}\left\|\eta_{\iota_{0}}\left(\lambda^{*}\right)-\eta_{\iota_{0}}\left(\lambda^{* *}\right)\right\|^{2},
\end{aligned}
$$

and therefore

$$
\begin{aligned}
& \mathbf{a}_{0}\left\|\pi_{x} V\left(y^{*}, \lambda^{*}\right)-\pi_{x} V\left(y^{* *}, \lambda^{* *}\right)\right\|^{2} \\
& <\min \left(-\mathbf{b}_{0},-\mathbf{c}_{0}\right)\left\|\left(y^{*}, \eta_{\iota_{0}}\left(\lambda^{*}\right)\right)-\left(y^{* *}, \eta_{\iota_{0}}\left(\lambda^{* *}\right)\right)\right\|^{2} .
\end{aligned}
$$

Now we move to proving the existence of the normally hyperbolic invariant manifold. First we need a definition.

Definition 12. We say that $f$ satisfies backward cone conditions if $f^{-1}$ satisfies cone conditions, with reversed roles of $x$ and $y$ coordinates.

We assume that for $f$ Assumption 7 holds with $\gamma_{0}=\gamma_{0}^{\text {forw }}$. We assume also that for $f^{-1}$ Assumption 7 holds with $\gamma_{0}=\gamma_{0}^{\text {back }}$ (with reversed roles of the $x$ and $y$ coordinates).

Theorem 13. (Main Theorem) Assume that $f$ satisfies cone conditions for $\gamma_{0}^{\text {forw }}=$ $\left(\mathbf{a}_{0}^{\mathrm{f}}, \mathbf{b}_{0}^{\mathrm{f}}, \mathbf{c}_{0}^{\mathrm{f}}\right)$ and backward cone conditions with $\boldsymbol{\gamma}_{0}^{\mathrm{back}}=\left(\mathbf{a}_{0}^{\mathrm{b}}, \mathbf{b}_{0}^{\mathrm{b}}, \mathbf{c}_{0}^{\mathrm{b}}\right)$. If

$$
\left|\mathbf{a}_{0}^{\mathrm{f}}\right|>\left|\mathbf{a}_{0}^{\mathrm{b}}\right| \text { and } \quad\left|\mathbf{b}_{0}^{\mathrm{f}}\right|<\left|\mathbf{b}_{0}^{\mathrm{b}}\right|
$$

then there exist continuous monomorphisms $W^{s}: B_{s}(0, r) \times \Lambda \rightarrow U, W^{u}: B_{u}(0, r) \times \Lambda \rightarrow$ $U$ and $\chi: \Lambda \rightarrow U$, such that

$$
\pi_{y, \lambda} W^{s}(y, \lambda)=(y, \lambda), \quad \pi_{x, \lambda} W^{u}(y, \lambda)=(x, \lambda), \quad \pi_{\lambda} \chi(\lambda)=\lambda,
$$


and $\Lambda_{\phi}:=\chi(\Lambda)$ is an invariant manifold for $f_{\phi}$, with stable manifold $W^{s}\left(B_{s}(0, r) \times \Lambda\right)$ and unstable manifold $W^{u}\left(B_{u}(0, r) \times \Lambda\right)$.

Proof. Since $f$ satisfies cone conditions, applying Theorem 11 we obtain $W^{s}(y, \lambda)$ as $V$. Since $f$ satisfies backward cone conditions, once again from Theorem 11 for $f^{-1}$ we also obtain $W^{u}(x, \lambda)$ as function $V$. From point i in Theorem 11 it follows that (25) holds for $W^{s}$ and $W^{u}$.

We shall show that for any $\lambda \in \Lambda$ the sets $W^{s}\left(B_{s}(0, r), \lambda\right)$ and $W^{u}\left(B_{u}(0, r), \lambda\right)$ intersect. Let us define $F: B_{u}(0, r) \times B_{s}(0, r) \rightarrow B_{u}(0, r) \times B_{s}(0, r)$ as

$$
F(x, y):=\left(\pi_{x} W^{s}(y, \lambda), \pi_{y} W^{u}(x, \lambda)\right) .
$$

Since $F$ is continuous, from the Brouwer fixed point theorem follows that there exists an $\left(x_{0}, y_{0}\right)$ such that $F\left(x_{0}, y_{0}\right)=\left(x_{0}, y_{0}\right)$. By (25) this means that

$$
W^{s}\left(y_{0}, \lambda\right)=\left(\pi_{x} W^{s}\left(y_{0}, \lambda\right), y_{0}, \lambda\right)=\left(x_{0}, \pi_{y} W^{u}\left(x_{0}, \lambda\right), \lambda\right)=W^{u}\left(x_{0}, \lambda\right) .
$$

Now we shall show that for any given $\lambda \in \Lambda$ there exists only a single point of such intersection. Suppose that for some $\lambda \in \Lambda$ there exist $\left(x^{*}, y^{*}\right),\left(x^{* *}, y^{* *}\right) \in$ $B_{u}(0, r) \times B_{s}(0, r),\left(x^{*}, y^{*}\right) \neq\left(x^{* *}, y^{* *}\right)$ such that

$$
W^{s}\left(y^{*}, \lambda\right)=W^{u}\left(x^{*}, \lambda\right) \text { and } W^{s}\left(y^{* *}, \lambda\right)=W^{u}\left(x^{* *}, \lambda\right) .
$$

From (25) we have $W^{s}\left(y_{m}, \lambda\right)=W^{u}\left(x_{m}, \lambda\right)=\left(x_{m}, y_{m}, \lambda\right)$ for $m=1,2$. From point 4 . in Theorem 11 follows that

$$
\begin{gathered}
Q_{\gamma_{0}^{\text {forw }}}\left(\tilde{\eta}_{i} \circ W^{s}\left(y^{*}, \lambda\right)-\tilde{\eta}_{i} \circ W^{s}\left(y^{* *}, \lambda\right)\right)= \\
Q_{\gamma_{0}^{\text {forw }}}\left(\left(x^{*}, y^{*}, \eta_{i}(\lambda)\right)-\left(x^{* *}, y^{* *}, \eta_{i}(\lambda)\right)\right)<0, \\
Q_{\gamma_{0}^{\text {back }}}\left(\tilde{\eta}_{i} \circ W^{u}\left(x^{*}, \lambda\right)-\tilde{\eta}_{i} \circ W^{u}\left(x^{* *}, \lambda\right)\right)= \\
Q_{\gamma_{0}^{\text {back }}}\left(\left(x^{*}, y^{*}, \eta_{i}(\lambda)\right)-\left(x^{* *}, y^{* *}, \eta_{i}(\lambda)\right)\right)<0 .
\end{gathered}
$$

which implies that

$$
\begin{aligned}
& a_{0}^{\mathrm{f}}\left\|x^{*}-x^{* *}\right\|^{2}+b_{0}^{\mathrm{f}}\left\|y^{*}-y^{* *}\right\|^{2}<0, \\
& a_{0}^{\mathrm{b}}\left\|x^{*}-x^{* *}\right\|^{2}+b_{0}^{\mathrm{b}}\left\|y^{*}-y^{* *}\right\|^{2}<0 .
\end{aligned}
$$

From (24) and (27) (keeping in mind that $a_{0}^{\mathrm{f}}>0, b_{0}^{\mathrm{f}}<0$ and that $a_{0}^{\mathrm{b}}<0, b_{0}^{\mathrm{b}}>0$ due to the reversion of the roles of $x$ and $y$ for the inverse map) follows that

$$
a_{0}^{\mathrm{f}}\left\|x^{*}-x^{* *}\right\|^{2}>-a_{0}^{\mathrm{b}}\left\|x^{*}-x^{* *}\right\|^{2}>b_{0}^{\mathrm{b}}\left\|y^{*}-y^{* *}\right\|^{2}>-b_{0}^{\mathrm{f}}\left\|y^{*}-y^{* *}\right\|^{2},
$$

which contradicts (26) .

We now define $\chi(\lambda):=\left(x_{0}, y_{0}, \lambda\right)$ for $x_{0}, y_{0}$ such that $W^{s}\left(y_{0}, \lambda\right)=W^{u}\left(x_{0}, \lambda\right)$. By above arguments we know that $\chi$ is a properly defined function. We need to show that this function is continuous. Let us take any $\lambda^{*}, \lambda^{* *} \in \eta_{i}^{-1}\left(B_{c}\left(\eta_{i}\left(\boldsymbol{\lambda}_{k}\right), \rho\right)\right)$ for some $(i, k) \in J$. From point 4 in Theorem 11 follows that

$$
\begin{aligned}
& Q_{\gamma_{0}^{\text {forw }}}\left(\tilde{\eta}_{i} \circ \chi\left(\lambda^{*}\right)-\tilde{\eta}_{i} \circ \chi\left(\lambda^{* *}\right)\right)<0, \\
& Q_{\gamma_{0}^{\text {back }}}\left(\tilde{\eta}_{i} \circ \chi\left(\lambda^{*}\right)-\tilde{\eta}_{i} \circ \chi\left(\lambda^{* *}\right)\right)<0 .
\end{aligned}
$$


Let us adopt notations $\tilde{\eta}_{i} \circ \chi\left(\lambda^{*}\right)=\left(x^{*}, y^{*}, \theta^{*}\right)$ and $\tilde{\eta}_{i} \circ \chi\left(\lambda^{* *}\right)=\left(x^{* *}, y^{* *}, \theta^{* *}\right)$. Note that from the construction of $\chi$ follows that $\eta_{i}\left(\lambda^{*}\right)=\theta^{*}$ and $\eta_{i}\left(\lambda^{* *}\right)=\theta^{* *}$. From (28) it follows that

$$
\begin{aligned}
& \left(a_{0}^{\mathrm{f}}+a_{0}^{\mathrm{b}}\right)\left\|x^{*}-x^{* *}\right\|^{2}+\left(b_{0}^{\mathrm{f}}+b_{0}^{\mathrm{b}}\right)\left\|y^{*}-y^{* *}\right\|^{2} \\
& <-\left(c_{0}^{\mathrm{f}}+c_{0}^{\mathrm{b}}\right)\left\|\theta^{*}-\theta^{* *}\right\|^{2} \\
& =-\left(c_{0}^{\mathrm{f}}+c_{0}^{\mathrm{b}}\right)\left\|\eta_{i}\left(\lambda^{*}\right)-\eta_{i}\left(\lambda^{* *}\right)\right\|^{2}
\end{aligned}
$$

From (24) it follows that $a_{0}^{\mathrm{f}}+a_{0}^{\mathrm{b}}=\left|a_{0}^{\mathrm{f}}\right|-\left|a_{0}^{\mathrm{b}}\right|>0$ and $b_{0}^{\mathrm{f}}+b_{0}^{\mathrm{b}}=-\left|b_{0}^{\mathrm{f}}\right|+\left|b_{0}^{\mathrm{b}}\right|>0$. By the fact that $\eta_{i}$ is continuous and the fact that $c_{0}^{\mathrm{f}}<0$ and $c_{0}^{\mathrm{b}}<0$, from (29) follows the continuity of $\chi$.

We will now show that for any $p \in W^{s}\left(B_{s}(0, r) \times \Lambda\right), f_{\phi}^{n}(p)$ converges to $\chi(\Lambda)$ as $n$ goes to infinity. Let us consider the limit set of the point $p$

$$
\omega\left(f_{\phi}, p\right)=\left\{q \mid \lim _{k \rightarrow \infty} f_{\phi}^{n_{k}}(p)=q \text { for some } n_{k} \rightarrow \infty\right\}
$$

If we can show that $\omega\left(f_{\phi}, p\right)$ is contained in $W^{u} \cap W^{s}=\chi(\Lambda)$, then this will conclude our proof. We take any $q=\lim _{k \rightarrow \infty} f_{\phi}^{n_{k}}(p)$ from $\omega\left(f_{\phi}, p\right)$. By continuity of $W^{s}$ we know that $q \in W^{s}$. Suppose now that $q \notin W^{u}$. This would mean that there exists an $n>0$ for which $f_{\phi}^{-n}(q) \notin B_{u}(0, r) \times B_{s}(0, r) \times \Lambda$. Since

$$
\lim _{k \rightarrow \infty} f_{\phi}^{n_{k}-n}(p)=f_{\phi}^{-n}(q)
$$

we have that $f_{\phi}^{-n}(q) \in \omega\left(f_{\phi}, p\right)$, but this contradicts the fact that $\omega\left(f_{\phi}, p\right) \subset B_{u}(0, r) \times$ $B_{s}(0, r) \times \Lambda$.

Showing that all backward iterations of points in $W^{u}\left(B_{u}(0, r) \times \Lambda\right)$ converge to $\chi(\Lambda)$ is analogous.

Remark 14. Let us note that during the course of the proof of Theorem 13 we have established more than just continuity of $W^{u}, W^{s}$ and $\chi$. From our construction we know that for $i \in I$

$$
\begin{aligned}
& \tilde{\eta}_{i} \circ W^{u}\left(x, \eta_{i}^{-1}(\theta)\right)=\left(x, w_{i}^{u}(x, \theta), \theta\right), \\
& \tilde{\eta}_{i} \circ W^{s}\left(y, \eta_{i}^{-1}(\theta)\right)=\left(w_{i}^{s}(y, \theta), y, \theta\right), \\
& \tilde{\eta}_{i} \circ \chi\left(\eta_{i}^{-1}(\theta)\right)=\left(\varkappa_{i}(\theta), \theta\right),
\end{aligned}
$$

for continuous $w_{i}^{u}: B_{u}(0, r) \times B_{c} \rightarrow B_{s}(0, r), w_{i}^{s}: B_{s}(0, r) \times B_{c} \rightarrow B_{u}(0, r)$ and $\varkappa_{i}: B_{c} \rightarrow B_{u}(0, r) \times B_{s}(0, r)$. The inequality (20) from Theorem 11 can be used to obtain explicit Lipschitz bounds for functions $w_{i}^{u}, w_{i}^{s}$. Also estimates (29) can be used to obtain Lipschitz bounds for $\varkappa_{i}$. This means that we can get Lipschitz estimates for the invariant manifold $\chi(\Lambda)$ together with Lipschitz estimates for its stable and unstable manifold. 


\section{Verification of covering and cone conditions}

In this section we show how covering relations and cone conditions can be verified with the use of local bounds on derivatives. The idea is to develop a simple automatised scheme which could be applied in computer assisted proofs. In our approach we set up our verification so that we do not need to compute images of large sets (which in case of rigorous numerics is always troublesome). The scheme is based on iterates of a number of single points, combined with estimates on derivatives around their neighbourhoods.

For any set $V \subset \mathbb{R}^{n}$ we define the interval enclosure of the derivative of $f$ on $V$ as

$$
\begin{aligned}
& {[d f(V)]:=} \\
& \left\{A \in \mathbb{R}^{n \times n} \mid A_{i j} \in\left[\inf _{x \in V} \frac{d f_{i}}{d x_{j}}(x), \sup _{x \in V} \frac{d f_{i}}{d x_{j}}(x)\right] \text { for all } i, j=1, \ldots, n\right\} .
\end{aligned}
$$

Let $U_{i_{1}}, U_{i_{2}} \subset \Lambda$ be such that $\operatorname{dom} f_{i_{2} i_{1}}$ is nonempty. Assume that for any $(c+u+s) \times$ $(c+u+s)$ matrix

$$
A \in\left[d f_{i_{2} i_{1}}\left(\operatorname{dom} f_{i_{2} i_{1}}\right)\right]
$$

we have the following bounds

$$
\begin{aligned}
& \sup \left\{\left\|A_{i j} v_{j}\right\|:\left\|v_{j}\right\|=1\right\} \leq \bar{A}_{i j} \\
& \inf \left\{\left\|A_{i j} v_{j}\right\|:\left\|v_{j}\right\|=1\right\} \geq \underline{A}_{i j},
\end{aligned}
$$

with $i, j \in\{1,2,3\}$ and $v_{1}, v_{2}, v_{3}$ representing the variables $x, y, \theta$ respectively (note that $\bar{A}_{i j}, \underline{A}_{i j}$ depend on the choice of $i_{2} i_{1}$ ). In this section we shall use the bounds (31) for verification of covering and cone conditions.

\subsection{Verifying covering conditions}

We define a $3 \times 3$ matrix $T_{i_{2} i_{1}}$ as

$$
\begin{array}{rlrl}
T_{i_{2} i_{1}} & :=\left(t_{i j}\right)_{i, j=1, \ldots, 3} \\
t_{11} & =\underline{A}_{11}, & t_{12}=-\bar{A}_{12}, & t_{13}=-\bar{A}_{13}, \\
t_{21}=\bar{A}_{21}, & t_{22}=\bar{A}_{22}, & t_{23}=\bar{A}_{23}, \\
t_{31}=\bar{A}_{31}, & t_{32}=\bar{A}_{32}, & t_{33}=\bar{A}_{33} .
\end{array}
$$

We will use notations $R=\left(r_{u}, r_{s}, r_{c}\right) \in \mathbb{R}^{3}$ and for $q=(x, y, \theta) \in \mathbb{R}^{u} \times \mathbb{R}^{s} \times \mathbb{R}^{c}$ and write

$$
N(q, R):=N\left(q, r_{u}, r_{s}, r_{c}\right)
$$

We give a lemma, which can be used in order to verify that $N_{1} \stackrel{f_{i_{i}}}{\longrightarrow} N_{2}$.

Lemma 15. Let $\varepsilon>0$ be a small number. Let $N_{1}=N\left(q_{1}, R_{1}\right) \subset \operatorname{dom} f_{i_{2} i_{1}}$ be a ch-set. If for $R_{2}=\left(r_{u}^{2}, r_{s}^{2}, r_{c}^{2}\right):=T_{i_{2} i_{1}} R_{1}+(-\varepsilon, \varepsilon, \varepsilon)$ we have $r_{u}^{2}, r_{s}^{2}, r_{c}^{2}>0$ and for $q_{2}:=f_{i_{2} i_{1}}\left(q_{1}\right)$

$$
\left\|\pi_{x} q_{2}\right\|+r_{u}^{2} \leq 1, \quad\left\|\pi_{y} q_{2}\right\|+r_{s}^{2} \leq 1, \quad\left\|\pi_{\theta} q_{2}\right\|+r_{c}^{2} \leq 1,
$$

then for $N_{2}:=N\left(q_{2}, R_{2}\right)$ we have $N_{1} \stackrel{f_{i_{2} i_{1}}}{\longrightarrow} N_{2}$. 
Proof. Condition (11) holds by the choice of $q_{2}$ and $N_{2}$. Let $q \in N_{1}^{-}$, then for

$$
A:=\int_{0}^{1} D f_{i_{2} i_{1}}\left(q_{1}+t\left(q-q_{1}\right)\right) d t \in\left[d f_{i_{2} i_{1}}\left(\operatorname{dom} f_{i_{2} i_{1}}\right)\right],
$$

we have estimates

$$
\begin{aligned}
\left\|\pi_{x}\left(f_{i_{2} i_{1}}(q)-q_{2}\right)\right\| & =\left\|\pi_{x}\left(f_{i_{2} i_{1}}(q)-f_{i_{2} i_{1}}\left(q_{1}\right)\right)\right\| \\
& =\left\|\pi_{x}\left(\int_{0}^{1} D f_{i_{2} i_{1}}\left(q_{1}+t\left(q-q_{1}\right)\right) d t \cdot\left(q-q_{1}\right)\right)\right\| \\
& =\left\|\pi_{x} A\left(q-q_{1}\right)\right\| \\
& =\left\|A_{11} \pi_{x}\left(q-q_{1}\right)+A_{12} \pi_{y}\left(q-q_{1}\right)+A_{13} \pi_{\theta}\left(q-q_{1}\right)\right\| \\
& \geq \underline{A}_{11} r_{u}^{1}-\bar{A}_{12} r_{s}^{1}-\bar{A}_{13} r_{c}^{1} \\
& >r_{u}^{2},
\end{aligned}
$$

hence (2) holds. Analogous computations for $q \in N_{1}$ give

$$
\begin{aligned}
\left\|\pi_{y}\left(f_{i_{2} i_{1}}(q)-q_{2}\right)\right\| & =\left\|\pi_{y} A\left(q-q_{1}\right)\right\| \leq \bar{A}_{21} r_{u}^{1}+\bar{A}_{22} r_{s}^{1}+\bar{A}_{23} r_{c}^{1}<r_{s}^{2}, \\
\left\|\pi_{\theta}\left(f_{i_{2} i_{1}}(q)-q_{2}\right)\right\| & =\left\|\pi_{\theta} A\left(q-q_{1}\right)\right\| \leq \bar{A}_{31} r_{u}^{1}+\bar{A}_{32} r_{s}^{1}+\bar{A}_{33} r_{c}^{1}<r_{c}^{2},
\end{aligned}
$$

which proves (3). Conditions (33) ensure that $N_{2} \subset \mathbf{B}$.

Example 16. We return to our Example 10, The ch-sets from the example follow from Lemma 9 as $N_{l}=N\left(0, R_{l}\right)$ where $R_{0}=(r, r)$ and $R_{l+1}=T_{i_{l+1} i_{l}} R_{l}+(-\varepsilon, \varepsilon, \varepsilon)$ with $T_{i_{l+1} i_{l}}=\operatorname{diag}\left(A_{11}^{l+1}, A_{22}^{l+1}\right)$.

Remark 17 . When the $x$ coordinate is strongly expanding, for practical reasons it might be beneficial to set $r_{u}^{2}$ significantly smaller than $\pi_{1} T_{i_{2} i_{1}} R_{1}$. In such case the covering $N_{1} \stackrel{f_{i_{2} i_{1}}}{\Longrightarrow} N_{2}$ will still take place, but $N_{2}$ will be a smaller set. This might give better bounds for next iterations of the map $f$ and also keep the later constructed $N_{i}$ within B. Without reducing $r_{u}$, in the case when $x$ is expanding, it might turn out that the sets $N_{i}$ blow up quickly.

\subsection{Verifying cone conditions}

Now we shall present some lemmas, which will show how one can obtain condition (17), from bounds on derivatives (31). The aim is to present a simple mechanism in which successive $\gamma_{l}$ are constructed.

Let $C=\left(c_{i j}\right)_{i, j=1, \ldots, 3}$ be a $3 \times 3$ matrix with coefficients

$$
\begin{array}{lll}
c_{11}=\underline{A}_{11}^{2}-\sum_{k \neq 1} \bar{A}_{11} \bar{A}_{1 k} & c_{12}=\sum_{k=1}^{3} \bar{A}_{21} \bar{A}_{2 k} & c_{13}=\sum_{k=1}^{3} \bar{A}_{31} \bar{A}_{3 k} \\
c_{21}=\underline{A}_{12}^{2}-\sum_{k \neq 2} \bar{A}_{12} \bar{A}_{1 k} & c_{22}=\sum_{k=1}^{3} \bar{A}_{22} \bar{A}_{2 k} & c_{23}=\sum_{k=1}^{3} \bar{A}_{32} \bar{A}_{3 k} \\
c_{31}=\underline{A}_{13}^{2}-\sum_{k \neq 3} \bar{A}_{13} \bar{A}_{1 k} & c_{32}=\sum_{k=1}^{3} \bar{A}_{23} \bar{A}_{2 k} & c_{33}=\sum_{k=1}^{3} \bar{A}_{33} \bar{A}_{3 k}
\end{array}
$$

(note that $C$ depends on the choice of $i_{2}, i_{1}$ ).

We start with a technical lemma 
Lemma 18. Let $\gamma=(a, b, c) \in \mathbb{R}^{3}$ and let $A: \mathbb{R}^{u+s+c} \rightarrow \mathbb{R}^{u+s+c}$ be a matrix for which the bounds (31) hold. If $a \geq 0, b \leq 0, c \leq 0$ then for any $p=\left(p_{1}, p_{2}, p_{3}\right) \in \mathbb{R}^{c} \times \mathbb{R}^{u} \times \mathbb{R}^{s}$

$$
Q_{\gamma}(A p) \geq Q_{C \gamma}(p) .
$$

Proof. Using the estimate

$$
\pm 2\left\langle A_{k i} p_{i}, A_{k j} p_{j}\right\rangle \geq-\bar{A}_{k i} \bar{A}_{k j}\left(\left\|p_{i}\right\|^{2}+\left\|p_{j}\right\|^{2}\right)
$$

we obtain

$$
\begin{aligned}
Q_{\gamma} & (A p) \\
= & a \sum_{i, j=1}^{3}\left\langle A_{1 i} p_{i}, A_{1 j} p_{j}\right\rangle+b \sum_{i, j=1}^{3}\left\langle A_{2 i} p_{i}, A_{2 j} p_{j}\right\rangle+c \sum_{i, j=1}^{3}\left\langle A_{3 i} p_{i}, A_{3 j} p_{j}\right\rangle \\
= & a \sum_{i=1}^{3}\left\|A_{1 i} p_{i}\right\|^{2}+b \sum_{i=1}^{3}\left\|A_{2 i} p_{i}\right\|^{2}+c \sum_{i=1}^{3}\left\|A_{3 i} p_{i}\right\|^{2} \\
& +2 \sum_{i<j} a\left\langle A_{1 i} p_{i}, A_{1 j} p_{j}\right\rangle+2 \sum_{i<j} b\left\langle A_{2 i} p_{i}, A_{2 j} p_{j}\right\rangle+2 \sum_{i<j} c\left\langle A_{3 i} p_{i}, A_{3 j} p_{j}\right\rangle \\
\geq & \left\|p_{1}\right\|^{2}\left(a \underline{A}_{11}^{2}+b \bar{A}_{21}^{2}+c \bar{A}_{31}^{2}\right)+\left\|p_{2}\right\|^{2}\left(a \underline{A}_{12}^{2}+b \bar{A}_{22}^{2}+c \bar{A}_{32}^{2}\right) \\
& +\left\|p_{3}\right\|^{2}\left(a \underline{A}_{13}^{2}+b \bar{A}_{23}^{2}+c \bar{A}_{33}^{2}\right) \\
& -a \sum_{i<j} \bar{A}_{1 i} \bar{A}_{1 j}\left(\left\|p_{i}\right\|^{2}+\left\|p_{j}\right\|^{2}\right)+b \sum_{i<j} \bar{A}_{2 i} \bar{A}_{2 j}\left(\left\|p_{i}\right\|^{2}+\left\|p_{j}\right\|^{2}\right) \\
& +c \sum_{i<j} \bar{A}_{3 i} \bar{A}_{3 j}\left(\left\|p_{i}\right\|^{2}+\left\|p_{j}\right\|^{2}\right) \\
= & (C \gamma)_{1}\left\|p_{1}\right\|^{2}+(C \gamma)_{2}\left\|p_{2}\right\|^{2}+(C \gamma)_{3}\left\|p_{2}\right\|^{2} .
\end{aligned}
$$

Now we give a lemma which will be the main tool in the construction of $\gamma_{l}$ from Definition 9 ,

Lemma 19. Let $U_{i_{1}}, U_{i_{2}} \subset \Lambda$ and let $N$ be a ch-set $N \subset \operatorname{dom}\left(f_{i_{2} i_{1}}\right)$. Let $\varepsilon>0$ be a small number. Let $C$ be defined by 34) and $\varepsilon>0$. Assume that $C$ is invertible and define

$$
G_{i_{2} i_{1}}=C^{-1} .
$$

If for $\gamma^{\prime}=(a, b, c):=G_{i_{2} i_{1}} \gamma+(\varepsilon, \varepsilon, \varepsilon)$, we have $a>0$, and $b, c<0$ then for any $q_{1}, q_{2} \in N$

$$
Q_{\gamma^{\prime}}\left(f_{i_{2} i_{1}}\left(q_{1}\right)-f_{i_{2} i_{1}}\left(q_{2}\right)\right)>Q_{\gamma}\left(q_{1}-q_{2}\right) .
$$

Proof. For

$$
A:=\int_{0}^{1} D f_{i_{2} i_{1}}\left(q_{2}+t\left(q_{1}-q_{2}\right)\right) d t \in\left[d f_{i_{2} i_{1}}\left(\operatorname{dom} f_{i_{2} i_{1}}\right)\right]
$$


applying Lemma 18 gives

$$
\begin{aligned}
Q_{\gamma^{\prime}}\left(f_{i_{2} i_{1}}\left(q_{1}\right)-f_{i_{2} i_{1}}\left(q_{2}\right)\right) & >Q_{G_{i_{2} i_{1}} \gamma}\left(f_{i_{2} i_{1}}\left(q_{1}\right)-f_{i_{2} i_{1}}\left(q_{2}\right)\right) \\
& \geq Q_{C G_{i_{2} i_{1}} \gamma}\left(q_{1}-q_{2}\right) \\
& =Q_{\gamma}\left(q_{1}-q_{2}\right) .
\end{aligned}
$$

Example 20. We return to Example 10, The cones $\gamma_{l}$ follow from Lemma 19 as $\gamma_{0}=(1,-1)$ and $\gamma_{l+1}=\left(1+\varepsilon,(1+\varepsilon)^{-1}\right) \cdot G_{i_{l+1} i_{l}} \gamma_{l}$ with $G_{i_{l+1} i_{l}}=\operatorname{diag}\left(\frac{1}{\left(A_{11}^{l+1}\right)^{2}}, \frac{1}{\left(A_{22}^{l+1}\right)^{2}}\right)$, where $\cdot$ stands for the scalar product.

\subsection{Setting up local maps}

In this section we shall introduce conditions, which would ensure that the assumptions from Section 3.2 hold. Below we give a Lemma which will ensure conditions (14) and (15).

Let us note that in some cases conditions (14) and (15) will follow from easier arguments or directly from the setup of the problem. Such is the case in our example from Section 5 ,

Lemma 21. Let $\mathbf{m}>1, \Delta>0$ and $\rho>\sqrt{\frac{\mathbf{a}_{0}}{-\mathbf{c}_{0}}} r+\Delta$. Assume that

(i) for any $\iota \in I$ and any $\lambda \in U_{\iota}$ there exists a $\boldsymbol{\lambda}_{\kappa}$ such that $(\iota, \kappa) \in J$ and

$$
\left\|\eta_{\iota}(\lambda)-\eta_{\iota}\left(\boldsymbol{\lambda}_{\kappa}\right)\right\|<\Delta
$$

(ii) for any $\theta \in B_{c}$ and any $i \in I$ there exists an $\iota \in I$ such that

$$
\begin{aligned}
& \bar{B}_{c}\left(\theta, \sqrt{\frac{\mathbf{a}_{1}}{-\mathbf{c}_{1}}} r\right) \cap \bar{B}_{c} \subset \operatorname{dom}\left(\eta_{\iota} \circ \eta_{i}^{-1}\right), \\
& \eta_{\iota} \circ \eta_{i}^{-1}(\theta) \in B_{c}(0, R-\rho-\Delta) .
\end{aligned}
$$

For $C_{\iota i}$ defined as in (34), constructed for $\left[d\left(\tilde{\eta}_{\iota} \circ \tilde{\eta}_{i}^{-1}\right)\left(\operatorname{dom}\left(\eta_{\iota} \circ \eta_{i}^{-1}\right)\right)\right]$ we assume that it is invertible and also that for $\gamma=(a, b, c)=C_{\iota i}^{-1} \gamma_{1}$ we have

$$
\mathbf{a}_{0}>\mathbf{m} a, \quad \mathbf{b}_{0}>\mathbf{m} b, \quad \mathbf{c}_{0}>\mathbf{m} c .
$$

If assumptions [2, ii hold, then for any horizontal disc $\mathbf{h}$ in a ch-set with cones $\left(M, \gamma_{1}\right)$ and for any $i \in I$ there exists $(\iota, \kappa) \in J$ such that $\mathbf{h}\left(\bar{B}_{u}(0, r)\right) \subset \operatorname{dom}\left(\tilde{\eta}_{\iota} \circ \tilde{\eta}_{i}^{-1}\right)$. Also for any $q_{1}, q_{2}$ in $\operatorname{dom}\left(\tilde{\eta}_{\iota} \circ \tilde{\eta}_{i}^{-1}\right)$ such that $Q_{\boldsymbol{\gamma}_{1}}\left(q_{1}-q_{2}\right)>0$ we have (14). Furthermore condition (15) holds.

Proof. Let $\mathbf{h}$ be a horizontal disc in a ch-set with cones $\left(M, \boldsymbol{\gamma}_{1}\right)$. Take $\theta_{0}=\pi_{\theta}(\mathbf{h}(0))$. For any $x \in \bar{B}_{u}(0, r)$ we have $Q_{\gamma_{1}}(\mathbf{h}(x)-\mathbf{h}(0)) \geq 0$, which implies that

$$
\mathbf{a}_{1} r^{2} \geq \mathbf{a}_{1}\left\|\pi_{x}(\mathbf{h}(x)-\mathbf{h}(0))\right\|^{2} \geq-\mathbf{c}_{1}\left\|\pi_{\theta}(\mathbf{h}(x))-\theta_{0}\right\|^{2}
$$


hence $\pi_{\theta}\left(\mathbf{h}\left(\bar{B}_{u}(0, r)\right)\right) \subset \bar{B}_{c}\left(\theta_{0}, \sqrt{\frac{\mathbf{a}_{1}}{-\mathbf{c}_{1}}} r\right) \cap \bar{B}_{c}$. Taking $\iota$ from assumption 2. for $\theta=\theta_{0}$, condition (37) implies that $\mathbf{h}\left(\bar{B}_{u}(0, r)\right) \subset \operatorname{dom}\left(\tilde{\eta}_{\iota} \circ \tilde{\eta}_{i}^{-1}\right)$ and also

$$
\left\|\eta_{\iota} \circ \eta_{i}^{-1}\left(\theta_{0}\right)\right\|<R-\rho-\Delta \text {. }
$$

Take now any $q_{1}, q_{2}$ in $\operatorname{dom}\left(\tilde{\eta}_{\iota} \circ \tilde{\eta}_{i}^{-1}\right)$ such that $Q_{\gamma_{1}}\left(q_{1}-q_{2}\right)>0$. Applying (39) and Lemma 19 gives

$$
\begin{aligned}
Q_{\gamma_{0}}\left(\tilde{\eta}_{\iota} \circ \tilde{\eta}_{i}^{-1}\left(q_{1}\right)-\tilde{\eta}_{\iota} \circ \tilde{\eta}_{i}^{-1}\left(q_{2}\right)\right) & >\mathbf{m} Q_{\gamma}\left(\tilde{\eta}_{\iota} \circ \tilde{\eta}_{i}^{-1}\left(q_{1}\right)-\tilde{\eta}_{\iota} \circ \tilde{\eta}_{i}^{-1}\left(q_{2}\right)\right) \\
& \geq \mathbf{m} Q_{C_{\iota} C_{\iota} C_{i} \gamma_{1}}\left(q_{1}-q_{2}\right) \\
& =\mathbf{m} Q_{\gamma_{1}}\left(q_{1}-q_{2}\right) \\
& >0,
\end{aligned}
$$

which proves (14). Applying the bound in (41) for $q_{1}=\mathbf{h}\left(x_{1}\right), q_{2}=\mathbf{h}\left(x_{2}\right)$ gives

$$
Q_{\gamma_{0}}\left(\mathbf{h}^{\prime}\left(x_{1}\right)-\mathbf{h}^{\prime}\left(x_{2}\right)\right) \geq 0,
$$

which means that to prove (15) it is sufficient to show that $\mathbf{h}^{\prime}\left(\bar{B}_{u}(0, r)\right) \subset M_{\iota, \kappa}$ for some $\kappa$. Let $\lambda=\eta_{i}^{-1}\left(\theta_{0}\right)$. We now take $\kappa$ from assumption 1 . For any $x \in \bar{B}_{u}(0, r)$, by (42) we have

$$
\begin{aligned}
\mathbf{a}_{0} r^{2} & \geq \mathbf{a}_{0}\left\|\pi_{x}\left(\mathbf{h}^{\prime}(x)-\mathbf{h}^{\prime}(0)\right)\right\|^{2} \\
& \geq-\mathbf{c}_{0}\left\|\pi_{\theta}\left(\mathbf{h}^{\prime}(x)-\mathbf{h}^{\prime}(0)\right)\right\|^{2} \\
& =-\mathbf{c}_{0}\left\|\pi_{\theta}\left(\mathbf{h}^{\prime}(x)\right)-\eta_{\iota}(\lambda)\right\|^{2} .
\end{aligned}
$$

This means that

$$
\pi_{\theta}\left(\mathbf{h}^{\prime}\left(\bar{B}_{u}(0, r)\right)\right) \subset \bar{B}_{c}\left(\eta_{\iota}(\lambda), r \sqrt{\frac{\mathbf{a}_{0}}{-\mathbf{c}_{0}}}\right)
$$

hence

$$
\begin{aligned}
\left\|\pi_{\theta}\left(\mathbf{h}^{\prime}(x)\right)-\eta_{\iota}\left(\boldsymbol{\lambda}_{\kappa}\right)\right\| & \leq\left\|\pi_{\theta}\left(\mathbf{h}^{\prime}(x)\right)-\eta_{\iota}(\lambda)\right\|+\left\|\eta_{\iota}(\lambda)-\eta_{\iota}\left(\boldsymbol{\lambda}_{\kappa}\right)\right\| \\
& <r \sqrt{\frac{\mathbf{a}_{0}}{-\mathbf{c}_{0}}}+\Delta \\
& <\rho,
\end{aligned}
$$

which gives $\mathbf{h}^{\prime}\left(\bar{B}_{u}(0, r)\right) \subset M_{\iota, \kappa}$. What needs to be verified last is whether $M_{\iota, \kappa} \subset \mathbf{B}$. From our construction $\pi_{\theta} M_{\iota, \kappa}=\bar{B}_{c}\left(\eta_{\iota}\left(\boldsymbol{\lambda}_{\kappa}\right), \rho\right)$. For $\theta \in \bar{B}_{c}\left(\eta_{\iota}\left(\boldsymbol{\lambda}_{\kappa}\right), \rho\right)$, using (36) and (40)

$$
\begin{aligned}
\|\theta\| & \leq\left\|\theta-\eta_{\iota}\left(\boldsymbol{\lambda}_{\kappa}\right)\right\|+\left\|\eta_{\iota}\left(\boldsymbol{\lambda}_{\kappa}\right)-\eta_{\iota}(\lambda)\right\|+\left\|\eta_{\iota}(\lambda)\right\| \\
& =\left\|\theta-\eta_{\iota}\left(\boldsymbol{\lambda}_{\kappa}\right)\right\|+\left\|\eta_{\iota}\left(\boldsymbol{\lambda}_{\kappa}\right)-\eta_{\iota}(\lambda)\right\|+\left\|\eta_{\iota} \circ \eta_{i}^{-1}\left(\theta_{0}\right)\right\| \\
& <\rho+\Delta+(R-\rho-\Delta)
\end{aligned}
$$

hence $\pi_{\theta} M_{\iota, \kappa} \subset B_{c}$. 


\subsection{Normally hyperbolic manifolds from bounds on derivatives}

In Section 4.1 we have shown how covering relations from the chain (16) can be constructed using bounds on derivatives of local maps. In Section 4.2 we have shown how the cones can be set up, using bounds on derivatives of local maps, so that the condition (17) holds. Here we shall combine these results together in Theorem 13 .

We shall use the notations $T_{i_{2} i_{1}}$ and $G_{i_{2} i_{1}}$ introduced in Sections 4.1, 4.2 through equations (32), (34) and (35). We will also assume that the assumptions from Section 3.2 hold. Here we introduce a definition which contains conditions which can be verified using computer assistance. We will later show that the conditions imply cone conditions.

Definition 22. Assume that for any $\left(\iota_{0}, \kappa_{0}\right) \in J$ there exists an $n \in \mathbb{N}$, a sequence $\iota_{0}=i_{0}, i_{1}, \ldots, i_{n}=\iota_{1}$ and $\kappa_{1}$ such that $\left(\iota_{1}, \kappa_{1}\right) \in J$ and for

$$
\begin{aligned}
& q^{m}=\left(x^{m}, y^{m}, \theta^{m}\right):=f_{i_{m} i_{m-1}} \circ \ldots \circ f_{i_{1} i_{0}}\left(0,0, \eta_{i_{0}}\left(\boldsymbol{\lambda}_{\kappa_{0}}\right)\right), \\
& R^{m}=\left(r_{u}^{m}, r_{s}^{m}, r_{c}^{m}\right):=T_{i_{m} i_{m-1}} \circ \ldots \circ T_{i_{1} i_{0}}(r, r, \rho), \\
& \gamma^{m}:=\left(a^{m}, b^{m}, c^{m}\right):=G_{i_{m} i_{m-1}} \circ \ldots \circ G_{i_{1} i_{0}} \gamma_{0}
\end{aligned}
$$

with $m \leq n$ we have

$$
\begin{array}{ll}
r_{u}^{m}+\left\|x^{m}\right\|<1, & r_{s}^{m}+\left\|y^{m}\right\|<1, \\
r_{u}^{n}>r+\left\|x^{n}\right\|, & r_{s}^{n}+\left\|y^{n}\right\|<r,
\end{array}
$$

and

$$
\begin{array}{lll}
a^{m}>0, & 0>b^{m}, & 0>c^{m}, \\
a^{n}>\mathbf{a}_{1}, & b^{n}>\mathbf{b}_{1}, & c^{n}>\mathbf{c}_{1} .
\end{array}
$$

Then we say that $f$ satisfies forward bounds.

Remark 23. To verify that $f$ satisfies forward bounds on needs to compute $q^{m}, R^{m}$ and $\gamma^{m}$. Let us note that in the case of $q^{m}$ it is enough to obtain bounds on a finite number of successive iterates of a single point. We therefore do not need to obtain bounds on images of large sets, which in practise would accumulate large errors. The $R^{m}$ and $\gamma^{m}$ are constructed using local bounds on derivatives and are easily computable with computer assistance. Let us also note that to verify forward bounds we do not need to compute the composition function $f^{n}$ or its derivative (this would most likely cause big difficulties for high $n$ due to complexity of such computations and also due to the fact that errors would accumulate quickly).

Lemma 24. If $f$ satisfies forward bounds then $f$ satisfies cone conditions.

Proof. We take any $\left(\iota_{0}, \kappa_{0}\right) \in J$, a sequence $\iota_{0}=i_{0}, i_{1}, \ldots, i_{n}=\iota_{1}$ and an index $\kappa_{1}$ such that $\left(\iota_{1}, \kappa_{1}\right) \in J$ from Definition 22. We define $R^{0}=R_{\varepsilon}^{0}:=(r, r, \rho)$ and

$$
\begin{aligned}
& R_{\varepsilon}^{m}:=T_{i_{m} i_{m-1}} R_{\varepsilon}^{m-1}+(-\varepsilon, \varepsilon, \varepsilon) \\
& N_{m}:=N\left(q^{m}, R_{\varepsilon}^{m}\right) .
\end{aligned}
$$

By (43), taking $\varepsilon$ sufficiently small, we will ensure that $N_{m} \subset$ B. By Lemma 15 we obtain $N_{m-1} \stackrel{f_{i_{m} i_{m}-1}}{\Longrightarrow} N_{m}$ for $m=1, \ldots, n$ and $N_{n} \stackrel{\text { id }}{\Longrightarrow} M$. 


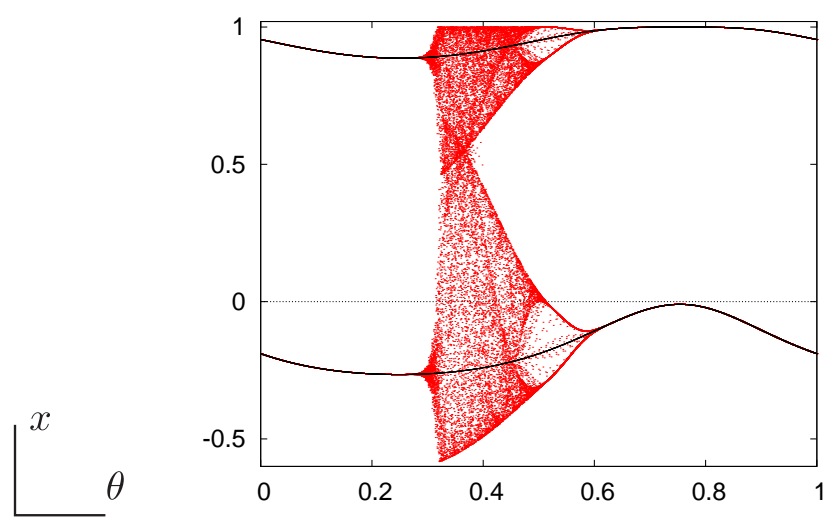

Figure 6. Misleading numerical plot of the attractor for $T$, obtained using double precision (grey), and the true invariant curve computed with 128bit accuracy (black).

Now we define $\gamma^{0}=\gamma_{\varepsilon}^{0}:=\gamma_{0}$ and

$$
\gamma_{\varepsilon}^{m}:=G_{i_{m} i_{m-1}} \gamma_{\varepsilon}^{m-1}+(\varepsilon, \varepsilon, \varepsilon) .
$$

Taking $\varepsilon>0$ small enough and applying Lemma 19 we obtain (17).

From now on let us assume that $f$ satisfies forward bounds with $\gamma_{0}=\gamma_{0}^{\text {forw }}$.

Definition 25. Let $\boldsymbol{\gamma}_{0}^{\text {back }}=\left(\mathbf{a}_{0}^{\mathrm{b}}, \mathbf{b}_{0}^{\mathrm{b}}, \mathbf{c}_{0}^{\mathrm{b}}\right) \in \mathbb{R}^{3}$ be such that $\mathbf{a}_{0}^{\mathrm{b}}, \mathbf{c}_{0}^{\mathrm{b}}<0$ and $\mathbf{b}_{0}^{\mathrm{b}}>0$. We say that $f$ satisfies backward bounds if $f^{-1}$ satisfies forward bounds, with reversed roles of the $x$ and $y$ coordinates.

Theorem 26. Assume that $f$ satisfies forward bounds for $\gamma_{0}^{\text {forw }}=\left(\mathbf{a}_{0}^{\mathrm{f}}, \mathbf{b}_{0}^{\mathrm{f}}, \mathbf{c}_{0}^{\mathrm{f}}\right)$ and backward bounds for $\boldsymbol{\gamma}_{0}^{\mathrm{back}}=\left(\mathbf{a}_{0}^{\mathrm{b}}, \mathbf{b}_{0}^{\mathrm{b}}, \mathbf{c}_{0}^{\mathrm{b}}\right)$. If in addition inequality 24) holds then there exists a normally hyperbolic invariant manifold in $\mathcal{U}$, together with its stable and unstable manifolds $W^{s}, W^{u}$.

Proof. This follows directly from Lamma 24 and Theorem 13.

\section{Example of applications}

Consider a driven logistic map

$$
\begin{aligned}
& T: S^{1} \times \mathbb{R} \rightarrow S^{1} \times \mathbb{R}, \\
& T(\theta, x)=\left(\theta+\alpha, 1-a(\theta) x^{2}\right), \quad a(\theta)=a_{0}+\varepsilon \sin (2 \pi \theta)
\end{aligned}
$$

which differs from the well-known logistic map in the fact that the parameter $a$ has been replaced by $a_{0}+\varepsilon \sin (2 \pi \theta)$ and $\theta$ has a quasiperiodic dynamics. Concretely we consider the parameter values $a_{0}=1.31, \varepsilon=0.3$ and $\alpha=\frac{g}{200}$, where $g$ is the golden mean $g=\frac{\sqrt{5}-1}{2}$, hence the dynamics on the base of the skew-product is slow. Numerical simulations in double precision (say, with mantissa of 52 binary digits) suggest that the map possesses a chaotic global attractor (see Figure 6, grey). We will prove that this guess is not correct. When the same simulations are done with multiple precision, one 
can guess that the attractor consists of two invariant curves (see Figure 6, black). We will use the method introduced in the previous sections to prove that $T$ possesses a contracting invariant manifold and, in particular, that the red plot from Figure 6 do not shows the true dynamics. The same example was considered for other values of $\alpha$ and in a non-rigorous way in [2] to illustrate that one has to be careful with the arithmetics in simulations.

\subsection{Explaining the observed behavior}

To explain the reasons of the observed behavior it is worth to mention that in the example the parameter $a$ of the logistic map ranges in $\left[a_{0}-\varepsilon, a_{0}+\varepsilon\right]=[1.01,1.61]$. For that range the attractor starts as a recently created (at $a=1$ ) period- 2 sink, followed by the full period-doubling cascade. Then one finds from several-pieces strange attractors to a single piece, interrupted by some periodic sinks and its corresponding cascades. When $a$ moves with $\theta$ one can question which is the "averaged" behavior. In particular the period-2 orbit is only attracting until $a=5 / 4$.

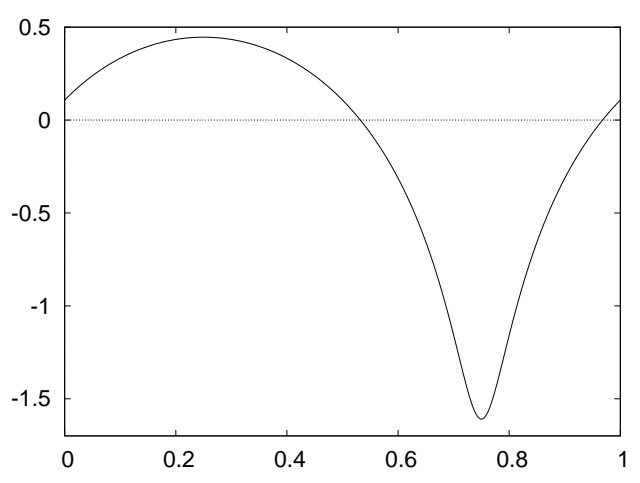

Figure 7. The integrand $h(\theta)$ in (46) for the parameter values: $a_{0}=1.31, \varepsilon=0.30$.

To this end we can consider what happens for "frozen" values of $a$, denoting as $T_{a}$ the corresponding logistic map. The orbit of period two, $x_{1}(a), x_{2}(a)$ is given by the solutions of $x^{2}-x / a+(1-a) / a^{2}$. In particular

$$
x_{1}(a)=(1-\sqrt{4 a-3}) /(2 a) .
$$

The differential of $T_{a}^{2}$ on it is $4(1-a)$. To average with respect to $\theta$ along the range, and noting that $a-1>0$ for the full range, we have to consider the average of the Lyapunov exponent given as

$$
\frac{1}{2} \int_{0}^{1} \log \left(4\left(a_{0}-1+\varepsilon \sin (2 \pi \theta)\right)\right) d \theta=\frac{1}{2} \log \left(2\left(a_{0}-1+\sqrt{\left(a_{0}-1\right)^{2}-\varepsilon^{2}}\right)\right)(
$$

which for $a_{0}=1.31, \varepsilon=0.3$ gives $\Lambda_{\infty} \approx-0.12666931$. The integrand is shown in Figure 7. For the skew product, assuming $\alpha \notin \mathbb{Q}$ and sufficiently small the two curves which form the attractor, as will be proved later, are very close to the curves $x_{1}(a), x_{2}(a)$ of the frozen system. Figure 8 displays the lower one. Also the Lyapunov exponent of the 
driven map with $\alpha=g / N, N=200$, computed using $10^{5}$ iterates after a transient also of $10^{5}$ iterates is $\Lambda_{200} \approx-0.12680$. Using other values of $N$, like $100,400,800,1600$ the respective values $\Lambda_{N}$ obtained are $-0.12725,-0.12670,-0.126696,-0.126689$, tending to the limit $\Lambda_{\infty}$.

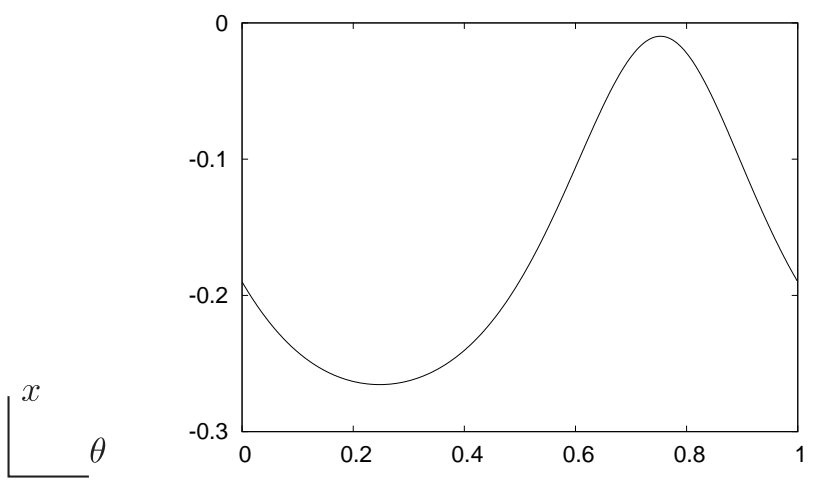

Figure 8. The lower part of the attractor, the graph of $x_{1}(a(\theta))$, for the parameter values: $a_{0}=1.31, \varepsilon=0.30$.

The numerical difficulties are easy to understand. To compute the Lyapunov exponents, starting at a point $x_{0}$ and an initial vector $v_{0}=1$ and setting $S_{0}=0$ we compute recurrently

$$
\begin{aligned}
& \hat{v}_{j+1}=D T_{a}\left(x_{j}\right)\left(v_{j}\right), \quad x_{j+1}=T_{a}\left(x_{j}\right), \quad n_{j+1}=\left|\hat{v}_{j+1}\right|, \quad v_{j+1}=\hat{v}_{j+1} / n_{j+1}, \\
& S_{j+1}=S_{j}+\log \left(n_{j+1}\right) .
\end{aligned}
$$

The values $S_{j}$ are denoted as Lyapunov sums and the average slope as a function of $j$ (if it exists) gives the Lyapunov exponent $\Lambda$. For details and generalisations see, e.g., [17] and [14] and references therein.

Even when $\Lambda$ is negative it can happen that partial sums have strong oscillations. Given the values of $S_{j}, j=0, \ldots, k$ let $\left(S_{k}\right)_{\text {min }}$ be the minimum of these values and introduce $O_{k}=S_{k}-\left(S_{k}\right)_{\min }$. We define the maximal oscillation of the Lyapunov sums as $O S=\max \left\{O_{k}\right\}$. The Figure 9 shows the behavior of $S_{j}$ for $\alpha=g / 200$ and also some of the initial oscillations for $\alpha=g / 1600$. A non-rigorous computation of $O S$ for $N=100,200,400,800,1600$ with $10^{5}$ iterates after a transient gives the values $28.845,56.761,112.632,224.379,447.874$, respectively. This implies a loss in the number of decimal digits equal to these values divided by $\log (10)$. In particular, between 24 and 25 digits for $N=200$, which explains the failure seen in Figure 6. For small $\alpha$ the maximal oscillation tends to be

$$
\frac{1}{\alpha} \int_{\theta_{2}-1}^{\theta_{1}} h(\theta) d \theta,
$$

where $h(\theta)$ is the function which appears as integrand in (46) and it is extended by periodicity outside $[0,1]$ while $\theta_{1}=\frac{3}{4}-\frac{1}{2 \pi} \cos ^{-1}(0.2), \theta_{2}=\frac{3}{4}+\frac{1}{2 \pi} \cos ^{-1}(0.2)$ are the values at which $h$ becomes equal to zero (see Figure 7$)$. The value of the maximal 
oscillation in (47) is $\approx 0.172660185 / \alpha$ for small $\alpha$, that if $\alpha=g / N$ becomes $\approx 0.27937 N$ in good agreement with the previous results.
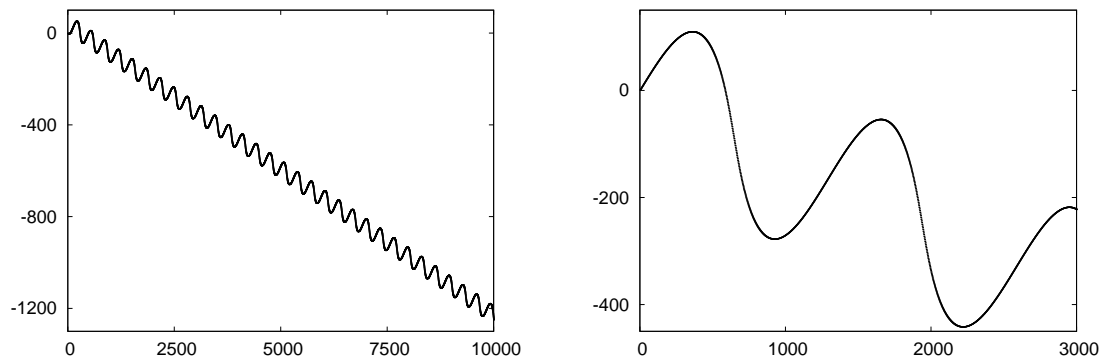

Figure 9. Oscillations of the Lyapunov sums. Left: the Lyapunov sums for $N=200$. Right: some initial sums for $N=1600$. Parameter values: $a_{0}=1.31, \varepsilon=0.30$ and $\alpha=g / N$.

Using these ideas one can even predict when we shall observe that the attractor produced by simulations with not enough digits seems to indicate that it is not a period2 curve. Assume that we do computations with $d$ decimal digits and that in a plot like the one in Figure 6 one can distinguish pixels which are a a distance of $10^{-p}$. In our example reasonable values of $d, p$ are 16 and 4 . This means that from $\theta_{2}-1$, when $h$ becomes positive, till some unknown $\theta_{d}$ when the "departure" of the iterates from the curve become visible, the factor of amplification of errors is $10^{d-p}$ or, in logarithmic scale $(d-p) \log (10)$. This requires

$$
\frac{1}{\alpha} \int_{\theta_{2}-1}^{\theta_{d}} h(\theta) d \theta=(d-p) \log (10) .
$$

In our example one finds $\theta_{d} \approx 0.258$ in good agreement with the observed numerics in Figure 6. In a similar way one can predict the "landing" value $\theta_{l}$ at which the points seen as chaotic in Figure 6 are close enough to the real invariant curves. As the distance from the chaotic points to the true attractor is of the order of 1 , the condition is now

$$
\frac{1}{\alpha} \int_{\theta_{1}}^{\theta_{l}} h(\theta) d \theta=p \log (10) .
$$

For the example one obtains $\theta_{l} \approx 0.629$, again in good agreement with the observed numerics.

This "delayed" observation of the expanding and compressing regimes is similar, but now due to purely numerical reasons, to the delay of bifurcation that can be observed in systems depending on a parameter which has slow dynamics (see [16] and references therein).

\subsection{Some limit cases}

Now we discuss two limit cases. First one is the case in which $a(\theta)$ covers a wide range. Second one aims at describing the differences between the union of the curves $x_{1}(a(\theta))$ and $x_{2}(a(\theta))$ and the true attractor for $\alpha$ small enough. 
According to (46) and assuming that for $\alpha$ sufficiently small the attractor is close to the union of the curves $x_{1,2}(a(\theta))$ it is enough to take $a_{0}=1.5-\delta_{1}, \varepsilon=0.5-\delta_{1}-\delta_{2}$ with $0<\delta_{2} \leq \delta_{1}^{2}$ to have a negative limit averaged Lyapunov exponent $\Lambda_{\infty}$. If $\delta_{1}$ is small the values of $a$ almost cover the full range $(1,2)$. The Figure 10] displays results of the observed behavior using double precision for the values $\delta_{1}=0.005, \delta_{2}=10^{-6}$, $\alpha=$ $g / 60000$.

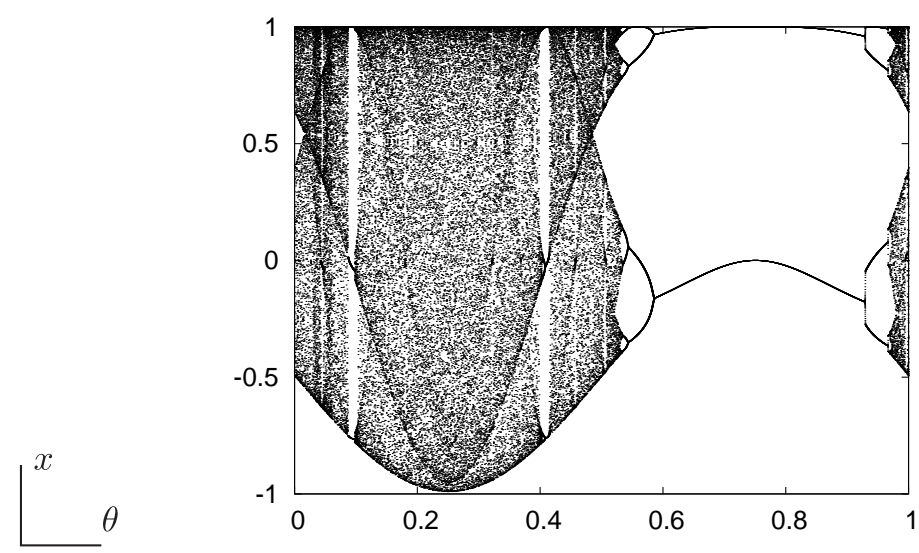

Figure 10. Simulations in double precision for values of $a_{0}, \varepsilon$ such that $a(\theta)$ almost covers the range $(1,2)$ and $\alpha$ very small. See the text for the numerical values used.

The figure is reminiscent of the "bifurcation diagram" of the logistic map. In fact, a typical way to compute the diagram consists of taking a sample of values of $a$, do some transient iterates and display some of the next iterates. Now the value of $a$ is changed at every step according to (44) but very slowly, and the transient is discarded. From $\theta=3 / 4$ (for which the minimum of $a(\theta)$ is achieved) to $\theta=5 / 4(\bmod 1)$ (for which one achieves the maximum) the plot looks like that diagram, except for the bifurcation delays at the period doublings from period 2 to period 4 and successive ones. In the range $\theta \in[1 / 4,3 / 4]$ the reverse situation is seen, but now with much smaller bifurcation delays. The authors do not know if, for the present values of the parameter, the attractor will become close to the union of $x_{1}(a(\theta))$ and $x_{2}(a(\theta))$ for computations done with a huge number of digits.

To look for the expression of the attractor as the union of two smooth curves, assuming it is of that type, we restrict our attention to the lower part of it, close to $x_{1}(a(\theta))$ as given in (45). In principle it is convenient to work with $T^{2}$ but, as the eigenvalues of $T^{2}$ along the points of period are negative, we prefer to work with $T^{4}$. We look for the attractor as the graph of a function expanded in powers of $\alpha$

$$
G(\theta)=G_{0}(\theta)+\alpha G_{1}(\theta)+\alpha^{2} G_{2}(\theta)+\ldots,
$$

where $G_{0}(\theta)=x_{1}(a(\theta))$ is the zeroth order approximation. The map $T^{4}(\theta, G(\theta))$ is $\mathcal{O}(\alpha)$ close to the identity. Hence, it can be approximated by a smooth flow (see [1] for proofs, an example of application and additional references, as well as [15] for general 
results) and the curve we are looking for is a periodic solution of this flow. But we shall proceed by imposing directly the invariance condition.

Starting at a point of the form $(\theta, G(\theta))$ and doing four iterations using the values $a(\theta), a(\theta+\alpha), a(\theta+2 \alpha), a(\theta+3 \alpha)$ we should have

$$
T^{4}(\theta, G(\theta))-(\theta+4 \alpha, G(\theta+4 \alpha))=0 .
$$

Given values of $a_{0}, \varepsilon$ it is a cumbersome but elementary task to obtain in a recurrent way the expressions of $G_{1}, G_{2}, \ldots$ from (49). It is essential to reduce the dependence in $G_{0}(\theta)$ using the equation satisfied by $x_{1}(a)$ to decrease the order of the powers of $G_{0}$ which appear to just the first one. We note also that in the computation of all the terms $G_{j}$ there appears $16 a^{2}-32 a+15=(4 a-5)(4 a-3)$ in the denominator, which cancels for $a=5 / 4$, but a careful examination allows to show that the factor $4 a-5$ is also present in the numerator.

In this way one obtains

$$
G_{1}(\theta)=\frac{3-2 a-(8 a-9) / \sqrt{4 a-3}}{2 a^{2}(4 a-3)} 2 \pi \varepsilon \cos (2 \pi \theta),
$$

where $a$ stands for $a(\theta)$ as introduced in (44).
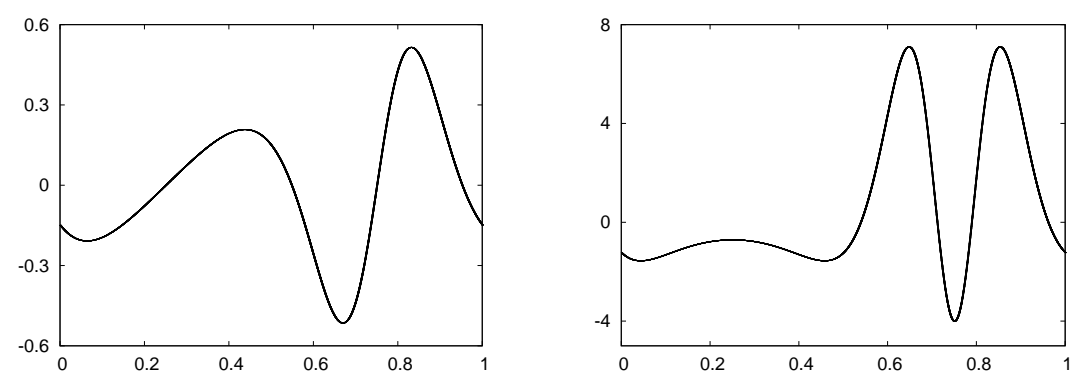

Figure 11. Graphs of $G_{1}(\theta)$ (left) and $G_{2}(\theta)$ (right) for $a_{0}=1.31, \varepsilon=0.30$.

The computation of $G_{2}$ is much more involved. The simplest expression is given as a rational function depending on $a(\theta), G_{0}(\theta), G_{1}(\theta)$ and up to the second derivatives of these functions with respect to $\theta$. Instead, Figure 11 displays the graph of $G_{1}$ and $G_{2}$ for $a_{0}=1.31, \varepsilon=0.30$. The graph of $G_{0}(\varepsilon)$ is very close to the attractor shown in Figure 8 .

To see tiny details on the attractor Figure 12 displays the differences between the lower part of the attractor, computed with enough digits, and the approximation in (48) up to order 2 in $\alpha$.

The left part shows tiny oscillations which were not visible in Figure 8, They reach a maximum at the value $\theta=\theta_{1}$ for which $h(\theta)$ in (47) changes from positive to negative. As one can expect the shape of these oscillations is a bump function multiplied by a periodic function (close to a sinus) with period $4 \alpha$. A similar behavior is observed for many other values of $a_{0}, \varepsilon$ and $\alpha$. When the oscillations start at a larger distance from $\theta_{1}$ they can amplify is such a way that the attractor is no longer the union of the two 

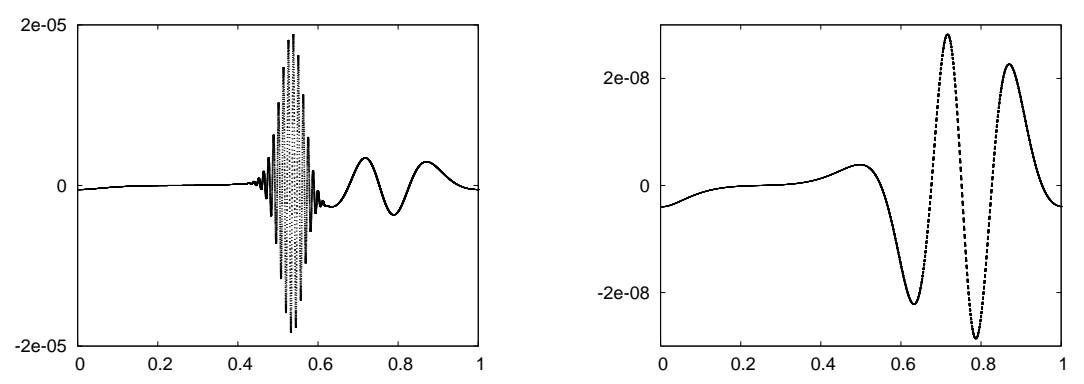

Figure 12. Differences between the attractor and the second order approximation for $a_{0}=1.31, \varepsilon=0.30$ and $\alpha=g / N$. Left: $N=200$. Right: $N=1000$.

curves. One can suspect that it becomes a non-chaotic strange attractor (see, e.g., 10] and [12]). In contrast, with the same values of $a_{0}, \varepsilon$ but for $N=1000$ the oscillations are not observed and the very small differences in the plot on the right hand side of Figure 12 are mainly due to the third order term in (48).

\subsection{Computer assisted proof of existence of invariant curves}

In this section we apply our method from Sections 3, 5.3.1 to prove that for parameters $a_{0}=1.31, \varepsilon=0.3$ and $\alpha=\frac{g}{200}$, with $g=\frac{\sqrt{5}-1}{2}$ the map $T$ has an invariant curve.

Around a neighborhood of the numerical guess for the attractor, the map $T^{2}$ is locally invertible. This is due to the fact that our curve is separated from the $x$-axis. For our proof we consider

$$
f=T^{-2} \text {. }
$$

The attractor is first computed (nonrigorously) by iterating $T$ forwards in time. We then choose a set $\mathcal{V}$ arround the attractor (see Figure 13, gray). For most $\theta$ the set is a 0.001 radius neighbourhood of the attractor. Close to the angle $\theta=\frac{3}{4}$ we choose $\mathcal{V}$ to be tighter, so that we are sure that it lies within the domain of $f$ (see Figure 13). Our aim is to prove that inside of $\mathcal{V}$ we have an invariant normally hyperbolic curve of $f$. The map $f$ is not uniformly expanding in the $x$ direction. Over one part of the set $\mathcal{V}$ the map $f$ is strongly expanding, elswehere it is contracting. A part of the expansion region, which we denote as $\mathcal{U} \subset \mathcal{V}$, is depicded in red and green (the green region is on the left tip of the red region and is poining towards the attractor) on Figure13, On this set we place ch-sets $N_{1}, \ldots, N_{168}$ of width $\frac{\alpha}{2}$, starting with $N_{1}$ on the left and finishing with $N_{168}$ on the right. We shall use a notation

$$
U_{k, l}=\bigcup_{i=k}^{l} N_{i}
$$

Our ch-sets are parallelograms. The coordinate $x$ is globally expanding for $f$ and coordinate $\theta$ is normal (our map does not posses a globally contracting coordinate $y$ ). The exits sets $N_{i}^{-}$for the ch-sets are the top and bottom edges of the parallelograms. The map $f$ moves the ch-sets to the left. We distinguish two parts of the set $\mathcal{U}$ : the set 


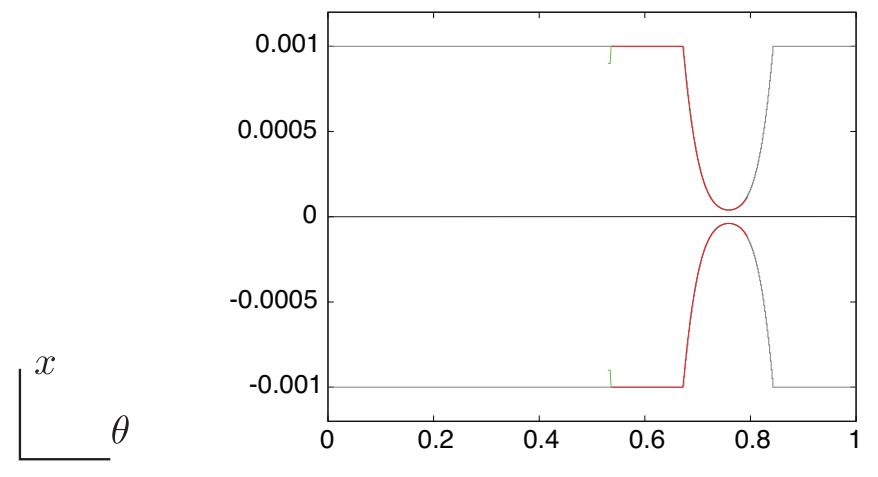

Figure 13. Positioning of our ch-sets (green and red) and the set $\mathcal{V}$ (gray) relative to the attractor (on this plot on the $\theta$-axis in black).
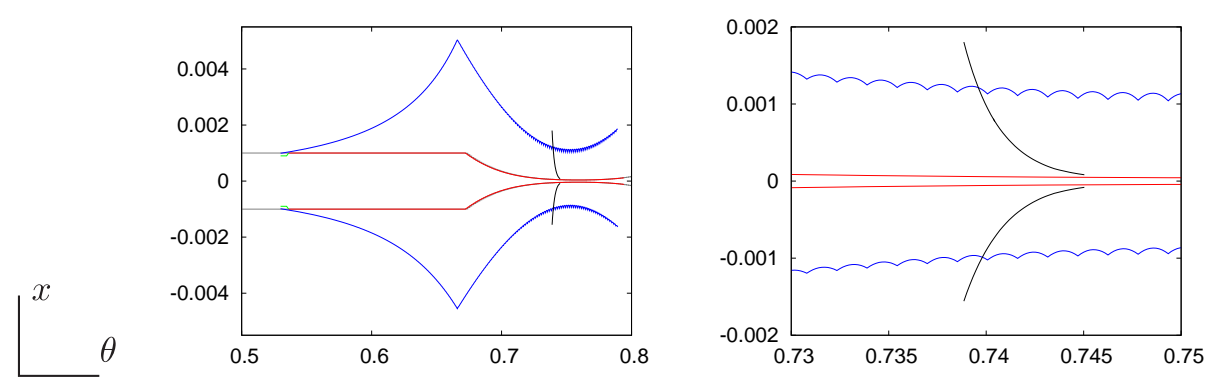

Figure 14. The ch-sets: $N_{1}^{-}, . . N_{4}^{-}$in green and $N_{5}^{-}, \ldots, N_{168}^{-}$in red (plotted relative to the attractor), together with $f\left(N_{5}^{-}\right), \ldots, f\left(N_{168}^{-}\right)$in dark blue and $f^{128}\left(N_{1}^{-}\right), \ldots, f^{128}\left(N_{4}^{-}\right)$in black.

$U_{1,4}$ in our plots is denoted in green colour, $U_{5,168}$ is denoted in red. Since the width of the ch-sets is $\frac{\alpha}{2}$, for $k \in 5, \ldots, 168$ we have

$$
\pi_{\theta} f\left(N_{k}\right) \subset \pi_{\theta} N_{k-4} .
$$

In Section 5.3.1 we shall show that (see Figures 14, 15)

$$
N_{k} \stackrel{f}{\Longrightarrow} N_{k-4} \text { for } k \in\{5, \ldots, 168\}
$$

and also that for $i=1, \ldots, 4$ (see Figure 14)

$$
N_{i} \stackrel{f^{128}}{\Longrightarrow} U_{5,168} \text {. }
$$

In Section 5.3 .2 we show how to verify cone conditions. In Section 5.3.3 we briefly discribe the tools that were used to conduct the proof.

5.3.1. Verification of covering conditions To describe how covering conditions are verified we start with a seemingly unrelated discussion. Consider a polynomial $p$ : $[0, r] \rightarrow \mathbb{R}$ of degree $n$

$$
p(\theta)=\sum_{j=0}^{n} a_{j} \theta^{j},
$$




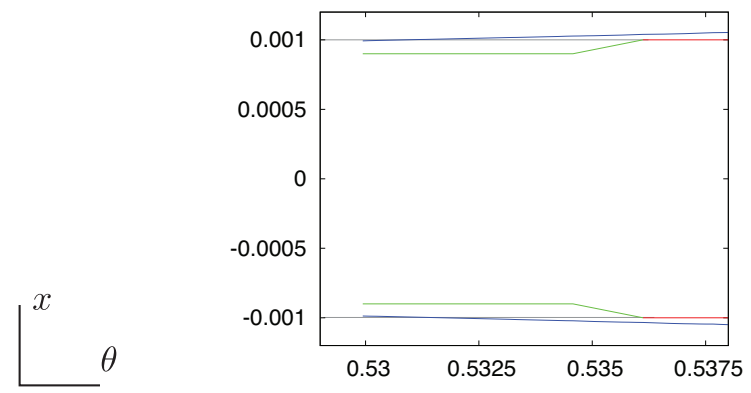

Figure 15. Closeup of the covering $N_{i} \stackrel{f}{\Longrightarrow} N_{i-4}$ for $i=5,6,7,8$ (plotted relative to the attractor).

and a function $g: \mathbb{R} \rightarrow \mathbb{R}$. Using Taylor expansion and defining two polynomials $\bar{p}$ and $\underline{p}$, of degree $n$

$$
\begin{aligned}
\bar{p}(\theta)= & g \circ p(0)+\sum_{j=1}^{n-1}\left(\frac{1}{j !} \frac{d^{j}(g \circ p)}{d \theta^{j}}(0)\right) \theta^{j} \\
& +\frac{1}{n !}\left(\frac{d^{n}(g \circ p)}{d \theta^{n}}(0)+\frac{1}{n+1} \sup _{v, w \in[0, r]} \frac{d^{n+1}(g \circ p)}{d \theta^{n+1}}(v) w\right) \theta^{n}, \\
\underline{p}(\theta)= & g \circ p(0)+\sum_{j=1}^{n-1}\left(\frac{1}{j !} \frac{d^{j}(g \circ p)}{d \theta^{j}}(0)\right) \theta^{j} \\
& +\frac{1}{n !}\left(\frac{d^{n}(g \circ p)}{d \theta^{n}}(0)+\frac{1}{n+1} \inf _{v, w \in[0, r]} \frac{d^{n+1}(g \circ p)}{d \theta^{n+1}}(v) w\right) \theta^{n},
\end{aligned}
$$

for any $\theta \in[0, r]$ we have

$$
\underline{p}(\theta) \leq g(p(\theta)) \leq \bar{p}(\theta) .
$$

For any $i=1, \ldots, 168$, the exit set $N_{i}^{-}$consists of two lines and can be expressed using two polynomials (in fact these are affine functions) $p_{i}^{u}, p_{i}^{d}:\left[0, \frac{\alpha}{2}\right] \rightarrow \mathbb{R}, p_{i}^{d}(\theta)=$ $a_{i, 0}^{d}+a_{i, 1}^{d} \theta, p_{i}^{u}(\theta)=a_{i, 0}^{u}+a_{i, 1}^{u} \theta$ and a point $q_{i} \in[0,1)$,

$$
\begin{aligned}
& N_{i}^{-}=N_{d}^{-} \cup N_{u}^{-}, \\
& N_{i, d}^{-}=\left\{\left(p_{i}^{d}(\theta), q_{i}+\theta\right) \mid \theta \in\left[0, \frac{\alpha}{2}\right]\right\}, \\
& N_{i, u}^{-}=\left\{\left(p_{i}^{u}(\theta), q_{i}+\theta\right) \mid \theta \in\left[0, \frac{\alpha}{2}\right]\right\}, \\
& p_{i}^{d}(\theta)<p_{i}^{u}(\theta) \quad \text { for } \theta \in\left[0, \frac{\alpha}{2}\right] .
\end{aligned}
$$

We will now show how to construct a ch-set $M$ such that

$$
N_{i} \stackrel{f}{\Longrightarrow} M \text {. }
$$

We first verify that for any point $(\theta, x) \in N_{i}$ we have $\frac{\partial f}{\partial x}(x, \theta)<0$. We then take

$$
g^{u}(\theta):=f\left(q_{i}+\theta, p_{i}^{d}(\theta)\right), \quad g^{d}(\theta):=f\left(q_{i}+\theta, p_{i}^{u}(\theta)\right),
$$


and construct $p^{u}(\theta)=\bar{p}(\theta)$ using (52) and $p^{d}(\theta)=\underline{p}(\theta)$ using (153), taking $g$ as functions $g^{u}$ and $g^{d}$ respectively. Formula (54) guarantees that $f\left(N_{i, d}^{-}\right)$lies above the graph of $p^{u}(\theta)$ and that $f\left(N_{i, u}^{-}\right)$lies below the graph of $p^{d}(\theta)$. If we now set

$$
\begin{aligned}
& M^{-}=M_{d}^{-} \cup M_{u}^{-}, \\
& M_{d}^{-}=\left\{\left(p^{d}(\theta), q_{i}-2 \alpha+\theta\right) \mid \theta \in\left[0, \frac{\alpha}{2}\right]\right\}, \\
& M_{u}^{-}=\left\{\left(p^{u}(\theta), q_{i}-2 \alpha+\theta\right) \mid \theta \in\left[0, \frac{\alpha}{2}\right]\right\},
\end{aligned}
$$

and take $M$ to be the set of points which lie above $M_{d}^{-}$and below $M_{u}^{-}$then (55) holds.

For $i=5, \ldots, 168$, after applying the above procedure to abtain $M$ which is covered by $N_{i}$, we compute bounds on the images of sets

$$
p^{u}\left(\left[\frac{j \alpha}{20}, \frac{(j+1) \alpha}{20}\right]\right), \quad p^{d}\left(\left[\frac{j \alpha}{20}, \frac{(j+1) \alpha}{20}\right]\right) \quad \text { for } j=0, \ldots, 9,
$$

in local coordinates of ch-sets $N_{i-4}$, to verify that we have (51) (subdividing $\left[0, \frac{\alpha}{2}\right]$ into ten intervals turns out to be sufficient for all $i \in\{5, \ldots, 168\})$.

For $i=1, \ldots, 4$ we need to iterate the procedure (57) many times to obtain a sequence of covering relations

$$
N_{i} \stackrel{f}{\Longrightarrow} M_{1} \stackrel{f}{\Longrightarrow} M_{2} \stackrel{f}{\Longrightarrow} \ldots \stackrel{f}{\Longrightarrow} M_{127} \stackrel{f}{\Longrightarrow} U_{5,168} .
$$

During our construction we make sure that all sets $M_{k}$ for $k \in\{1, \ldots, 127\}$ lie in $\mathcal{V}$, which readily holds since the sets are very strongly contracted. Each covering $M_{k} \stackrel{f}{\Longrightarrow} M_{k+1}$ holds by construction. Verifying that $M_{127} \stackrel{f}{\Longrightarrow} U_{5,168}$ is done analogously to (58).

In our computer assisted proof we take the degrees of polynomials for the edges of the sets $M_{k}$ as nine, which means that we need to perform $C^{10}$ computations. Let us note that computationally this is not as heavy as might seem, since the $C^{10}$ computations are performed for one dimensional functions $g^{u}(\theta)$ and $g^{d}(\theta)$ (see (56) ). The reduction of dimension truly pays off, since the difference between $C^{10}$ computations in one and two dimensions is substantial.

The estimates obtained by us are very accurate. In Figure 16 we give a plot of $M_{128, u}^{-}$, which is the lower bound estimate of the image of $N_{4, u}^{-}$after the final step in our procedure (in black), and compare it with ten points from $N_{4, u}^{-}$, iterated nonrigorously with high precision computations (in red). The curve lies below the points, as should, but this is impossible to distinguish from the graph. The right hand side of Figure 16 gives the plot of the difference of the rigorous lower bound and non-rigorous computation. They turn out to be very close.

Remark 27. The high order computations and multi-precision in current approach seem essential. The sets $M_{k}$ constructed with our procedure are very strongly contracted. The distance between the two curves of $M_{k}^{-}$at the tightest spot is of order $1.125 \times 10^{-25}$, which is extremely thin when compared to the width of the curves $\frac{\alpha}{2} \approx 1.545 \times 10^{-3}$; and yet, with our $C^{10}$ approach, with little effort we are able to rigorously keep them apart. Any standard approach, such as performing $C^{0}$ computations on sets or careful linearization with $C^{1}$ techniques through local coordinates, is likely to fail. 


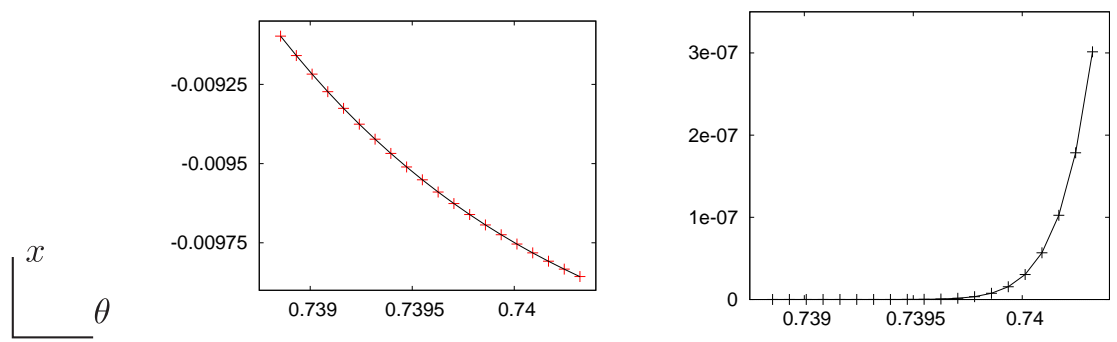

Figure 16. Left: Rigorous bound on the image of an edge of one of ch-sets after 128th iterate of the map (in black), together with non-rigorous computations using multiprecision (red). Right: The difference between rigorous lower bound and non-rigorous computations.

Remark 28. We believe that using a "parallel shooting" type approach it should be possible to conduct the proof using double precision and $C^{1}$ computations only (for this we would need an good apriori guess for the position of the curve). Such approach could produce a rigorous-computer-assisted proof using double precision of an invariant a curve, which is not detectable numerically with double computations. This shall be a subject of forthcoming work.

5.3.2. Verification of cone conditions To verify cone conditions let us first rescale our coordinates by

$$
\gamma_{\beta}(\theta, x)=(\beta \theta, x) .
$$

Taking $\beta$ sufficiently large, choosing sufficiently many points $\lambda_{i} \in[0, \beta)$ and taking $h_{i}:=\frac{1}{2}\left(c^{u}\left(\lambda_{i}\right)-c^{d}\left(\lambda_{i}\right)\right), q_{i}:=\left(\lambda_{i}, c^{d}\left(\lambda_{i}\right)+h_{i}\right)$ and $V_{i}:=\mathcal{V} \cap\left(\left[\lambda_{i}-h_{i}, \lambda_{i}+h_{i}\right] \times \mathbb{R}\right)$ we can construct local maps

$$
\tilde{\eta}_{i}: V_{i} \rightarrow B_{c} \times B_{u},
$$

for which $\tilde{\eta}_{i}\left(V_{i} \cap c^{u}\right)=B_{c} \times\{1\}, \tilde{\eta}_{i}\left(V_{i} \cap c^{d}\right)=B_{c} \times\{-1\}$ and which are arbitrarily close to a linear map $q \rightarrow \frac{1}{h_{i}}\left(q-q_{i}\right)$. In these local coordinates, by taking sufficiently large $\beta$, we have the following bound on derivatives of local maps (assuming that we choose $i, j$ and $p$ such that $\left.p \in \operatorname{dom}\left(f_{i j}\right) \neq \emptyset\right)$

$$
\begin{aligned}
D f_{i j}= & D\left(\tilde{\eta}_{i} \circ \gamma_{\beta} \circ f \circ \gamma_{\beta}^{-1} \circ \tilde{\eta}_{j}^{-1}\right)(p) \\
& \approx\left(\begin{array}{cc}
\frac{1}{h_{i}} & 0 \\
0 & \frac{1}{h_{i}}
\end{array}\right)\left(\begin{array}{cc}
\beta & 0 \\
0 & 1
\end{array}\right)\left(\begin{array}{cc}
\frac{d f_{1}}{d \theta}\left(\gamma_{\beta}^{-1}\left(\tilde{\eta}_{j}^{-1}(p)\right)\right. & 0 \\
\frac{d f_{2}}{d \theta}\left(\gamma_{\beta}^{-1}\left(\tilde{\eta}_{j}^{-1}(p)\right)\right. & \frac{d f_{2}}{d x}\left(\gamma_{\beta}^{-1}\left(\tilde{\eta}_{j}^{-1}(p)\right)\right.
\end{array}\right) \\
& \left(\begin{array}{cc}
\beta^{-1} & 0 \\
0 & 1
\end{array}\right)\left(\begin{array}{cc}
h_{j} & 0 \\
0 & h_{j}
\end{array}\right) \\
= & \frac{h_{j}}{h_{i}}\left(\begin{array}{cc}
\frac{d f_{1}}{d \theta}\left(\gamma_{\beta}^{-1}\left(\tilde{\eta}_{j}^{-1}(p)\right)\right. \\
\frac{1}{\beta} \frac{d f_{2}}{d \theta}\left(\gamma_{\beta}^{-1}\left(\tilde{\eta}_{j}^{-1}(p)\right)\right. & \frac{d f_{2}}{d x}\left(\gamma_{\beta}^{-1}\left(\tilde{\eta}_{j}^{-1}(p)\right)\right.
\end{array}\right),
\end{aligned}
$$


which in turn is arbitrarily close to $\frac{h_{j}}{h_{i}} \operatorname{diag}\left(\frac{d f_{1}}{d \theta}, \frac{d f_{2}}{d x}\right)$. This means that by using the artificial rescaling $\gamma_{\beta}$ (without the actual need to apply it in practice for our computer assisted proof), we can divide the region $\mathcal{V}$ into a finite number of sets $U_{1}, \ldots, U_{N}\left(\mathcal{V} \subset \bigcup_{i=1}^{N} U_{i}\right)$, and verify cone conditions using interval matrices $\operatorname{diag}\left(\left[\frac{d f_{1}}{d \theta}\left(U_{i}\right)\right],\left[\frac{d f_{2}}{d x}\left(U_{i}\right)\right]\right)$ and applying Lemma 19. For our proof we take $\gamma_{0}=(a, b)=(1,-1)$, which means that the quadratic form for our cones is simply

$$
Q_{\gamma_{0}}(\theta, x)=x^{2}-\theta^{2} \text {. }
$$

If we take $\gamma_{1}=((1-\varepsilon), 1)$ for any small parameter $\varepsilon>0$ then by choosing sufficiently large $\beta$ Assumption 7 is satisfied (since any switch to new coordinates is arbitrarily close to identity). This means that we can take $\gamma_{1}=\gamma_{0}$, provided that all the inequalities in our verification of cone conditions in the computer assisted proof are strict.

5.3.3. Tools used for the proof Our proof has been conducted with the use of the CAPD library (http://capd.ii.uj.edu.pl) developed by the Computer Assisted Proofs in Dynamics group. We have used the multi-precision version of the library running at 128 mantisa bits accuracy (which is approximately equivelent to tracking 40 digits). The $C^{10}$ computations have been performed with assistance of the Flexible Automatic Differentiation Package FADBAD++ (www.fadbad.com). The proof takes 16 seconds running on a $2.53 \mathrm{GHz}$ laptop with $4 \mathrm{~GB}$ of RAM.

\section{Final comments}

In this paper we have presented a version of a normally hyperbolic invariant manifold theorem, which can be applied for rigorous-computer-assisted proofs. We have successfully applied our method to an example in which standard double precision simulations brake down and produce false results. This demonstrates the strength of our method, that it can handle numerically difficult cases. It needs to be noted that to apply our method we have used multiple precision for our computer assisted computations. For our proof we also needed to apply a high order method which relied on $C^{10}$ computations. We believe that it should be possible to devise a similar in spirit method, which would give proofs without multiple precision and using $C^{1}$ computations only. This will be the subject of our future work.

\section{Acknowledgements}

We would like to thank Tomasz Kapela for his assistence and comments regarding the implementation of multi-precision in CAPD library. Our special thanks goes to Daniel Wilczak for his suggestions, frequent discussions and for his assistence with implementation of higher order computations in the CAPD library. The research of MC has been supported by the Polish State Ministry of Science and Information Technology grant N201 543238. The research of CS has been supported by grants MTM200605849/Consolider (Spain), and CIRIT 2008SGR-67 (Catalonia). 


\section{References}

[1] H. Broer, R. Roussarie, C. Simó. Invariant circles in the Bogdanov-Takens bifurcation for diffeomorphisms, Ergod. Th. \& Dynam. Sys. 16 (1996), 1147-1172.

[2] H. Broer, C. Simó, R. Vitolo, Chaos and quasi-periodicity in diffeomorphisms of the solid torus, DCDS B 14 (2010), 871-905.

[3] M. J. Capiński, Covering Relations and the Existence of Topologically Normally Hyperbolic Invariant Sets, Discrete and Continuous Dynamical Systems A. Vol. 23, N. 3, (March 2009), pp 705-725

[4] M. J. Capiński, P. Roldán, Existence of a Center Manifold in a Practical Domain around $L_{1}$ in the Restricted Three Body Problem, to appear in SIAM J. on Appl. Dyn. Sys.

[5] M. J. Capiński P. Zgliczyński, Cone Conditions and Covering Relations for Normally Hyperbolic Invariant Manifolds, preprint

[6] M. J. Capiński and P. Zgliczyński, Covering Relations and Non-autonomous Perturbations of ODEs, Discrete Contin. Dyn. Syst. Ser. A, 14, 281-293 (2006).

[7] Z. Galias, Positive topological entropy of Chua's circuit: A computer assisted proof. Int. J. Bifurcation and Chaos, 7(2):331-349, 1997.

[8] Z. Galias and P. Zgliczyński, Computer assisted proof of chaos in the Lorenz system, Physica D, 115, 1998, 165-188.

[9] M. Gidea and P. Zgliczyński, Covering relations for multidimensional dynamical systems, J. Differential Equations , 202(2004) 33-58.

[10] C. Grebogi, E. Ott, S. Pelikan and J. A. Yorke, Strange attractors that are not chaotic, Physica D 13 (1984), 261-268.

[11] M. Hirsh, C. Pugh and M. Shub, Invariant Manifolds, Lecture Notes in Mathematics, Vol. 583. Springer-Verlag, Berlin-New York, 1977.

[12] T. Jäger, On the structure of strange non-chaotic attractors in pinched skew products, Ergodic Theory and Dynamical Systems 27 (2007), 493-510.

[13] C. K. R. T. Jones, Geometric singular perturbation theory. Dynamical systems (Montecatini Terme, 1994), 44-118, Lecture Notes in Math., 1609, Springer, Berlin, 1995.

[14] F. Ledrappier, M. Shub, C. Simó, A. Wilkinson, Random versus deterministic exponents in a rich family of diffeomorphisms, J. Stat Phys. 113 (2003), 85-149.

[15] A.I. Neishtadt, The separation of motions in systems with rapidly rotating phase, Prikladnaja Matematika i Mekhanika 48 (1984), 133-139.

[16] A. I. Neishtadt, C. Simó, D. V. Treschev, On stability loss delay for a periodic trajectory, in Progress in nonlinear differential equations and their applications. Vol 19, pp. 253-278, Birkhauser-Verlag, Basel, Switzerland, 1995.

[17] C. Simó, Global Dynamics and Fast Indicators, in Global Analysis of Dynamical Systems, H. W. Broer, B. Krauskopf, G. Vegter, editors, pp. 373-390, IOP Publishing, Bristol, 2001.

[18] D. Wilczak, Chaos in the Kuramoto-Sivashinsky equations - a computer assisted proof, J. Differential Equations 194 (2003), no. 2, 433-459.

[19] P. Zgliczyński, Covering relations, cone conditions and stable manifold theorem J. of Diff. Equations 246 (2009) 1774-1819, 